\title{
PRENATAL DEVELOPMENT OF FUNCTIONAL CONNECTIONS IN THE CAT'S RETINOGENICULATE PATHWAY ${ }^{1}$
}

\author{
CARLA J. SHATZ AND PETER A. KIRKWOOD ${ }^{2}$ \\ Department of Neurobiology, Stanford Universily School of Medicine, Stanford, California 94305 \\ Received September 2, 1983; Revised December 2, 1983; Accepted December 2, 1983
}

\begin{abstract}
The development of functional connections between the axons of retinal ganglion cells and the neurons of the dorsal lateral geniculate nucleus (LGNd) of fetal and neonatal cats was studied using an in vitro assay. Extracellular microelectrode recordings of single units were made from histologically identified sites in the LGNd of isolated diencephalon preparations between embryonic day 39 (E39) and postnatal day 2 (P2). (Gestation is 65 days in the cat.) Postsynaptic units activated by electrical stimulation of one or both optic nerves were found at all ages tested from E39 onwards. Over $90 \%$ of the units studied in the fetal preparations received convergent excitation from both optic nerves, compared with roughly half of the units studied in the neonatal preparations. Inhibition was detected in the LGNd of the neonatal preparations, but in only the oldest of the fetal preparations (E59). This physiological change from predominantly convergent excitation to an adult-like mixture of excitation and inhibition seen at birth coincides with the change from mixed to segregated afferent input from the two eyes seen anatomically (Shatz, C. J. (1983) J. Neurosci. 3: 482-499). These results indicate that attainment of the adult pattern of retinogeniculate connectivity involves the elimination of already functional synapses.
\end{abstract}

During the development of the vertebrate nervous system, the orderly adult pattern of afferent input to a target structure is often absent initially. Rather, the final pattern of connectivity emerges secondarily during a period in which originally intermixed inputs gradually segregate from each other. Over the past several years, many examples have been reported in which this segregation process occurs during development-in both the peripheral (Brown et al., 1976; Lichtman and Purves, 1980; Johnson and Purves, 1981) and central (Crepel et al., 1976; Rakic, 1976; LeVay et al., 1978, 1980; So et al., 1978; Mariani and Changeux, 1981a, b; review by Purves and Lichtman, 1980) nervous systems. Perhaps the most graphic example is that of the development of the mammalian visual system, in which inputs from the two eyes

\footnotetext{
${ }^{1}$ We wish to thank all members of the Shatz Laboratory for their help with the fetal surgery, Mark Siegel for his assistance with the photography, and Cecele Thomas for typing. We are grateful to Dr. M. P. Stryker for his criticisms of the manuscript. This work could not have been performed without assistance from the National Institutes of Health (Grant EYO2858) and the McKnight Foundation to C. J. S. P. A. K. was Alexander Piggot Werhner Memorial Trust Fellow of the Medical Research Council, on leave from the Institute of Neurology, London.

${ }^{2}$ Present address: Sobell Department of Neurophysiology, Institute of Neurology, National Hospital, Queen Square, London WC1N 3BG, England.
}

are initially intermixed with each other within both the lateral geniculate nucleus (LGN) and the primary visual cortex, and later sort out from each other to achieve the familiar laminar (LGN) or columnar (cortex) organization characteristic of the adult (Rakic, 1976; LeVay et al., 1978, 1980; So et al., 1978; Linden et al., 1981; Bunt et al., 1983; Shatz, 1983).

It is not known how this remarkable transformation from a mixed to a segregated state comes about during development. However, evidence from studies so far indicates that the transformation occurs during a period in which axonal inputs destined to segregate from each other are first capable of forming functioning synaptic connections with common postsynaptic target neurons (for review see Purves and Lichtman, 1980). Such evidence underlies a current popular hypothesis that segregation is achieved via an activity-related competitive process requiring the formation and also the elimination of synaptic connections (Stent, 1973; Changeux and Danchin, 1976; Purves and Lichtman, 1980).

In order to investigate this hypothesis further, we chose to study the development of connections between retinal ganglion cells and their target neurons within the LGN of the cat. This choice was made because the cat's LGN is the site of convergence of two distinct and experimentally separable inputs from the two eyes. In the adult cat, axons arising from the retinal ganglion 
cells of each eye terminate in alternate layers within the LGN. It is now known that this pattern of termination is established prenatally (Shatz, 1983): retinal afferents from the two eyes are initially intermixed within the LGN. Then, beginning around embryonic day 47 (E47), the two sets of afferents segregate from each other to give rise to the adult-like laminar pattern of input achieved by birth, 3 weeks later. Furthermore, electron microscopic studies in progress have indicated that retinal ganglion cell axons form synapses within the LGN throughout the segregation period and even as early as E43, prior to its onset (Shatz et al., 1982; C. J. Shatz and M. W. Siegel, manuscript in preparation). Therefore, the question arises whether before and during segregation, functioning connections are present between both sets of retinal afferents and common postsynaptic LGN neurons.

To begin to answer this question, we have developed an in vitro thalamus preparation to assess electrophysiologically the functional state of connections between retina and LGN from fetal cats of known gestational ages between E39 and birth. We have made extracellular microelectrode recordings from single LGN units in this preparation in order to study their responses to electrical stimulation of the optic nerves. Our findings demonstrate that, throughout the transformation of retinal input from a mixed to a segregated state, connections between the segregating inputs and the postsynaptic target neurons are functional. They further suggest that connections are operational within the mammalian visual system throughout a large period of fetal development-many weeks before the system is put to the use for which it is ultimately designed. Some of these results have been reported previously in preliminary form (Shatz et al., 1982; Kirkwood and Shatz, 1983).

\section{Materials and Methods}

Animals used and surgery. Twenty normal cat fetuses and three normal neonatal kittens were studied. Ages of fetuses ranged from embryonic day 39 (E39) to E59 (the cat's gestation period is approximately 65 days). Fetuses were delivered by Cesarean section from mother cats under halothane $(0.5$ to $1.5 \%)$ and nitrous oxide and oxygen (1:2) anesthesia and placed on ice. All but two of the fetuses described here were from timed pregnancies and their embryonic ages were therefore known to within $\pm 24 \mathrm{hr}$. (The time of first mating was designated as E0 and the day of birth as P0.) The ages of the other two (littermates) were estimated from crown-rump length, the general morphology of the brain, and the detailed histological appearance of the thalamus (in particular the relative positions of the lateral and medial geniculate nuclei). These estimated ages were accurate to within \pm $48 \mathrm{hr}$. Full details of the surgery and determination of the embryonic ages are given in Shatz (1983).

The three neonatal kittens were anesthetized with halothane and nitrous oxide and oxygen via a face mask and were also placed on ice. Ages of these kittens were: postnatal day 2 (P2), P1, and estimated P0 (this kitten was born prematurely following surgery on the mother cat 3 days previously).

In six fetuses and one neonatal kitten, the vitreous body of one eye was injected with $\left[{ }^{3} \mathrm{H}\right]$ leucine 1 or 2 days before the physiological experiment, as described by Shatz (1983), to facilitate the histological identification of the LGN and the recording sites.

In vitro preparation. Apart from the anesthesia, which was maintained in the kittens until the removal of the entire diencephalon, the dissection procedure for the kittens and the fetuses was identical. First, enucleation was performed bilaterally, leaving most of the optic nerve in the orbit. Next, the top half of the cranium was removed and most of the telencephalon was carefully lifted away from the diencephalon. At all ages the positions of the lateral and medial geniculate bodies were obvious as two clear bumps on the dorsolateral surface of the exposed diencephalon. The lower part of the skull was then trimmed to isolate an approximately rectangular piece of bone at the base of the skull, attached to the brain around the optic foramina. The diencephalon and some brainstem were then removed with this piece of bone and placed in ice-cold Ringer's solution of the following composition: $\mathrm{NaCl}, 124 \mathrm{~mm} ; \mathrm{KCl}, 5 \mathrm{~mm}$; $\mathrm{KH}_{2} \mathrm{PO}_{4}, 1.15 \mathrm{mM} ; \mathrm{MgSO}_{4} \cdot 7 \mathrm{H}_{2} \mathrm{O}, 1.15 \mathrm{mM} ; \mathrm{CaCl}_{2}, 2.5$ $\mathrm{mm} ; \mathrm{NaHCO}_{3,2} 25 \mathrm{~mm}$; D-glucose, $10 \mathrm{~mm}$; bubbled with $95 \% \mathrm{O}_{2}, 5 \% \mathrm{CO}_{2}$ (Crepel et al., 1981). This solution was also used to bathe the exposed brain during the earlier dissection.

The remaining dissection was carried out in the cold Ringer's solution. The bone was removed from around the optic nerves so as to leave the nerves as long as possible for stimulation. This procedure was not always successful, and one or another nerve was often rather short and sometimes damaged. In a good dissection from the youngest fetuses, 1 to $2 \mathrm{~mm}$ of each nerve were retained; 2 to $3 \mathrm{~mm}$ were retained in the older ones. From the dorsal side of the preparation a sagittal cut was made into the third ventricle, leaving only the optic chiasm, the base of the hypothalamus, and the pons intact. Finally the preparation was spread out and pinned ventral side up onto a wedge of Sylgaard 184 (DowCorning Ltd.) which was already glued onto a thin plastic platform $(8 \mathrm{~mm} \times 24 \mathrm{~mm})$. In this inverted, spread out position both the optic nerves and the LGN were now conveniently accessible from above. The platform with the preparation attached was then mounted on a Plexiglas ring and placed in an "Oslo type" tissue slice chamber with modifications similar to those of Dhanjal and Sears (see Dingledine, 1984, appendix). Oxygenated Ringer's solution (as above) flowed at a rate of 1 to $2 \mathrm{ml} /$ min around the preparation from underneath and was sucked away to waste. The preparation was totally submerged, with the optic chiasm (and most of the optic tract) less than $1 \mathrm{~mm}$ below the surface. Warmed, humidified gas $\left(95 \% \mathrm{O}_{2}, 5 \% \mathrm{CO}_{2}\right)$ flowed over the surface and the temperature was maintained at $32^{\circ} \mathrm{C}\left( \pm 1^{\circ} \mathrm{C}\right)$. The time taken from delivery or anesthesia to immersion in the slice chamber was typically around $1 \mathrm{hr}$, of which about $45 \mathrm{~min}$ were spent in the in vitro dissection. Preparations from fetuses older than E55 or from the neonatal kittens seemed to survive well only if dissection time was less than $45 \mathrm{~min}$.

Stimulation and recording. In order to assess the functional state of connections between retina and LGN, 
electrical stimuli were delivered to the two optic nerves and extracellular microelectrode recordings were made from the LGN. The optic nerves were separately stimulated with rectangular electrical pulses, delivered via simple suction electrodes filled with Ringer's solution. To minimize stimulus spread, the outer contact (cathode) was a wire wrapped around the suction tube and arranged to enter the bathing medium on the side of the electrode farthest from the other nerve but not too close to the optic tract. In a few experiments, when damage or impaired conduction in both nerves at the region of the optic chiasm was suspected, the optic tract itself was dissected and placed in a suction electrode. The volleys evoked by optic nerve or tract stimulation were routinely monitored by means of a silver ball electrode insulated except on its lower surface and placed on the optic tract roughly midway between the chiasm and the LGN from which microelectrode recordings were made.

Recordings from the optic tract were essential for successful experiments. They allowed the condition of the preparation to be assessed, the afferent inputs to be monitored, and stimulus spread from the electrode on one nerve to the fibers of the other nerve to be detected. For example, Figure $1 A$ shows maximal volleys recorded at one site in the optic tract from stimulation of each of the two nerves. Volley amplitudes and waveforms are typical of preparations in good condition. Volleys with complex waveforms were frequently seen with stimulation of the contralateral nerve (most often a double negative wave, here a triple negative wave). We do not believe this complex waveform to be the result of damage for several reasons. First, the same waveforms could be seen when the volleys were recorded from the other optic tract (i.e., a single negative peak from the ipsilateral nerve but a double peak from the contralateral nerve). Moreover, the same configuration was observed in the optic tracts of neonatal rats in vitro (C. J. Shatz and P. A. Kirkwood, unpublished results) where the nerves are very easy to dissect and unlikely to be damaged, and where it was shown that the two negative peaks represent different conduction velocities of contralateral fibers by moving the recording electrode along the tract. Finally, in neither rats nor cats could the two components be readily separated by varying stimulus strength.

The amplitudes of the volleys were indications of the state of the preparation, the most successful experiments having volleys greater than $200 \mu \mathrm{V}$ peak to peak. However, a better indication was the stability of volley size. In the older animals (E55 or above) volleys recorded within $10 \mathrm{~min}$ of putting the preparation into the recording chamber usually declined in amplitude to almost zero within another $10 \mathrm{~min}$ or so. Then, in successful experiments at these ages, the volleys recovered in amplitude within about an hour, as has been reported for the electrical activity of most slice preparations (Schwartzkroin, 1981). Volleys that recovered more slowly usually declined in amplitude again within 1 or $2 \mathrm{hr}$, and even when they were fairly large, the postsynaptic activity in these preparations was always less than that in other preparations of comparable ages. Of the 20 fetuses studied, six preparations (all between E55 and E63) gave only small or unstable volleys, and observations from these
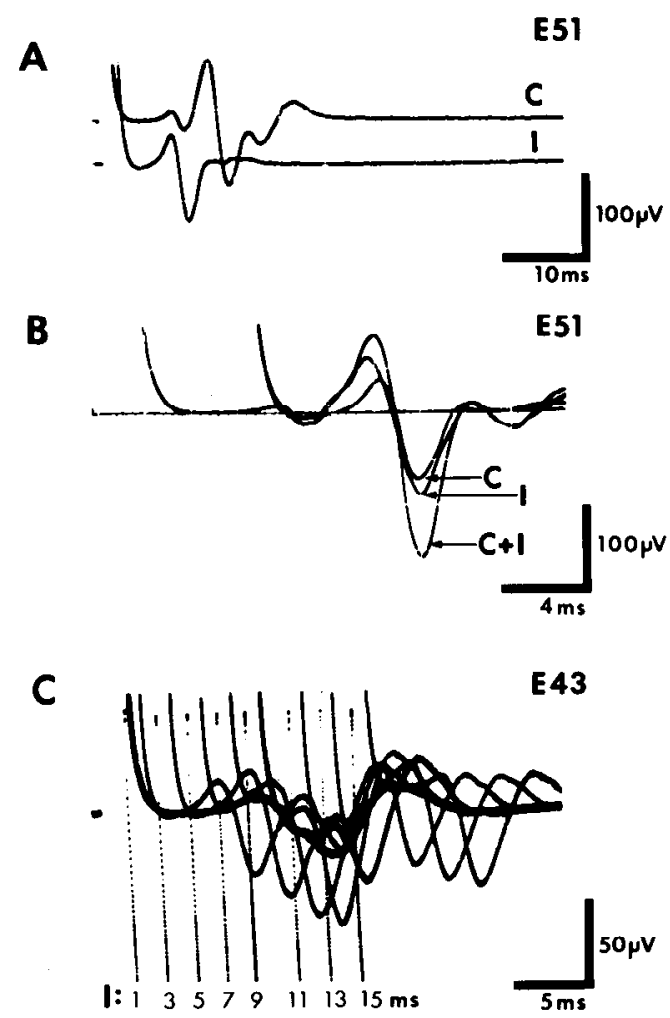

Figure 1. Volleys recorded from the optic tract. $A$, Volleys from the contralateral and ipsilateral nerves of an E51 fetus stimulated separately at the same delay on each trace. $B$, Refractoriness test for stimulus spread in this E51 preparation. Contralateral $(C)$ and ipsilateral $(I)$ volleys were stimulated separately at different delays so that the main negative peaks of the volley were superimposed. These two stimuli were then given together $(C+I)$. The traces $C$ and $I$ add algebraically to equal $C+I$, indicating absence of stimulus spread. $C$, The same procedure as $B$ but in an E43 fetus, and with the time interval between the two stimuli varied as indicated below the stimulus artifacts from the ipsilateral stimuli. The response to the contralateral stimulus alone is represented by the heavy line; the responses to the various combinations are represented by the other traces. At all intervals, the responses to the two stimuli added linearly, again indicating absence of stimulus spread. In this and all other figures, positive is upward. See "Materials Methods" for further details.

are excluded from the results that follow. Some of the other experiments (mostly on younger fetuses) also gave small volleys, but results are included because the volleys were stable or even increased slowly during the experiment. (In the younger fetuses an initial period of deep depression of electrical activity was not seen.) In three fetuses in which the volleys from nerve stimulation were small and decreased with time, the tract was dissected and stimulated directly. Gradual failure of the volley usually occurred after many hours of recording (up to 15 hr) and was taken as the criterion to end the experiment. The amplitude of the volleys was also a good indicator of the degree of oxygenation of the preparation.

Nerves in good condition commonly gave volley thresholds of about 10 to $15 \mathrm{~V}$ and maximum volley amplitudes at around 50 to $70 \mathrm{~V}$ for stimulus durations varying between $0.2 \mathrm{msec}$ (for the oldest animals) and $0.5 \mathrm{msec}$ (for the youngest animals). Note that the stim- 
ulus voltages used, although high, are not exceptional given the diameters of nerve fibers involved $(0.1 \mu \mathrm{m}$ to $0.6 \mu \mathrm{m}$ at E48; Williams et al. 1983) and the stimulus durations selected (Gasser, 1950). Nerves that were very short (possibly damaged) required higher stimulus strengths, with thresholds and maximal values up to twice as great.

In all preparations, but especially in those with higher volley thresholds, the possibility of stimulus spread from one electrode to the other nerve was a serious concern, particularly since one of our major goals was to compare the postsynaptic responses of single LGN units evoked by stimulation of each optic nerve. Therefore, a refractoriness test to assess the extent of stimulus spread was routinely employed at the beginning of each experiment and also following single unit recordings. The test was based on the following reasoning: If stimulating current is indeed spreading from one electrode to the other nerve, then it should be possible to stimulate some of the fibers in that nerve from both electrodes. Hence, at some time interval, fibers stimulated by one electrode should be refractory to stimulation via the other. Occlusion should therefore occur between the optic tract volleys evoked from stimulation of the two nerves. This was indeed observed in some preparations, particularly when the nerves were very short and stimulus strengths above 80 $\mathrm{V}$ were used. On the other hand, if stimulus spread is not a problem, then the volley amplitude evoked to combined stimulation of the two nerves should always equal the algebraic sum of the volleys evoked to each stimulus separately. Examples of this are shown in Figure $1, B$ and $C$. In Figure $1 B$, the same preparation as that of Figure $1 A$ (E51), the stimulus time separation (6 msec) was chosen to make the two main negative peaks evoked by stimulation of the contralateral $(C)$ and ipsilateral $(I)$ nerves coincide in time (Fig. $1 B$, arrows). Figure $1 C$ shows another example (E43) in which a range of stimulus separations was chosen. This is the range (1 to $15 \mathrm{msec}$ ) we routinely used in testing for stimulus spread. In neither of these two cases, nor in three others, (E59, P0, and $\mathrm{P} 2$ ) was there any occlusion, even at maximal stimulus strengths. In two additional animals (E49 and E54) occlusion was present, but only at maximal stimulus strengths (see Table II and "Results" for further details). In four of the animals stimulus spread was a serious concern, and these were excluded from any analysis of the convergence of inputs from the two nerves. In the remaining six animals, only one nerve or the tract could be stimulated.

A low stimulus rate was chosen in order not to fatigue immature synapses. Single shocks were delivered to the nerves at a rate of 0.2 stimulus/sec, a compromise to allow us to obtain a reasonable sample of cells. In some of the youngest fetuses this compromise was adjusted downward and rates of 0.1 or 0.15 stimulus/sec were used.

Either very sharp tungsten microelectrodes (Hubel, 1957; Shatz, 1977) or glass ones (resistance 10 to 30 megohms, filled with $2 \mathrm{M} \mathrm{NaCl}$ ) were used for extracellular recording of unit spikes. A short time constant was almost always selected on the recording amplifier. Electrode tracks, in particular important recording sites, were marked by electrolytic lesions via the tungsten electrodes using currents of 5 to $7 \mu \mathrm{A}$ for 5 to $7 \mathrm{sec}$. Sites of recording from the glass electrodes were calculated from the positions of parallel tracks marked with lesions by tungsten electrodes, and these positions were only accepted as accurate if the entry points of the two tracks were within $100 \mu \mathrm{m}$ of each other, the penetrations were short, and no dimpling of the surface was observed.

Histology. At the end of each experiment, the preparation pinned to its platform was fixed overnight by immersion in $4 \%$ paraformaldehyde, $5 \%$ sucrose in $0.1 \mathrm{M}$ phosphate buffer. After an additional $36 \mathrm{hr}$ in the same buffered fixative with $20 \%$ sucrose added, frozen sections were cut at 30 or $40 \mu \mathrm{m}$, mounted on subbed slides, prepared for autoradiography if required (Shatz, 1983), and stained with cresyl violet for electrode track reconstruction.

To facilitate reconstructions, the plane of section was usually chosen to be parallel to the electrode tracks. Since the electrodes were directed perpendicular to the platform base, sections were cut roughly $45^{\circ}$ from the normal parasagittal planes, as indicated on the inset to Figure 2. Figure 2 shows such a section from an E43 fetus stained with cresyl violet that contained an electrode track marked by two lesions (Fig. 2, arrows). Clear identification of electrode tracks was important because we wished to know in particular whether recording sites were located in the dorsal or ventral lateral geniculate nucleus (LGNd or LGNv). As shown in Figure 2, the distinction is straightforward even at this early age and in this unusual plane of section. It was usually also possible to distinguish the medial interlaminar nucleus (MIN) (not present in this section), and in all fetuses it could be determined that no recordings were from sites in the MIN.

An additional histological feature associated with these in vitro experiments is illustrated in Figure 2: centered near the deeper lesion (arrow) is an area of necrosis characterized by the presence of darkly stained, shrunken cells. Necrotic regions, presumably the result of anoxia, were found in all but the most superficial sections of every preparation studied (see also Figs. $9 A$ and $18 A$ ). Nevertheless, it was possible accasionally to record postsynaptic activity within these areas either because a few healthy neurons remained (as appeared to be the case histologically), or because recordings were made sufficiently early during the experiment prior to the onset of extensive damage.

Table I shows the location of all of the postsynaptic units (see "Results" for criteria) recorded in the 17 successful experiments. Of the 119 postsynaptic single units recorded in this study, $74(62 \%)$ were located unambiguously within the dorsal LGN and these are the subject of the present report.

\section{Results}

Procedures for the assessment of postsynaptic responses. Before considering in systematic detail the responses recorded from neonatal and fetal isolated thalamus preparations studied in vitro, it is worth illustrating several general features of the postsynaptic responses 


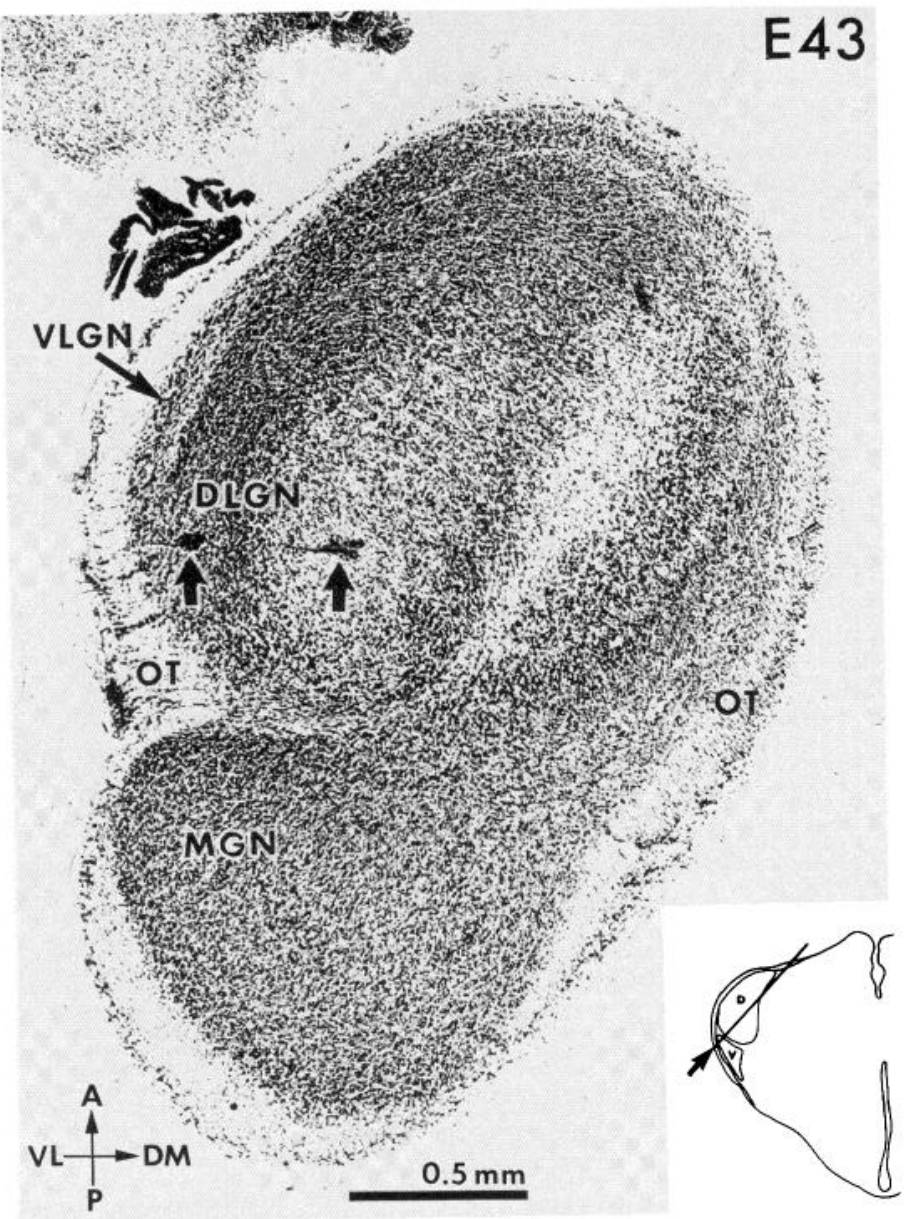

Figure 2. Cresyl violet-stained section containing an electrode track and two lesions from an E43 preparation. Large arrows show the lesions along the track which stays entirely within LGNd after passing through the optic tract $(O T)$. In this plane of section, the LGNv is a thin wafer along the ventrolateral margin of the thalamus. $M G N$, medial geniculate nucleus. Inset, The approximate plane of section is shown in a drawing from a more familiar coronal section of another E43 fetus. $D$, LGNd; $V$, LGNv; arrow, point of entry of electrode.

and the identification criteria used to distinguish extracellularly recorded pre- and postsynaptic activity. In all preparations, single-unit spikes were recorded with various amplitudes (up to 2 or $3 \mathrm{mV}$ ). Spike shapes were most often diphasic or triphasic, or sometimes more complex (e.g., Fig. 6). The total duration, even of simple diphasic spikes, was often long $(3 \mathrm{msec})$. In a typical experiment, we alternated between glass and tungsten microelectrodes in recording from the LGN. Glass electrodes gave us the best signal-to-noise ratio, but tungsten electrodes allowed us to record multiunit activity which was of great use in assessing the general areas where activity could be found and the state of the preparation. For example, Figure $3 A$ shows a multiunit recording from a site in the LGNd of a young fetus (E43). (The general features of this recording are typical of those performed at all ages.) A single shock to the contralateral nerve elicited a multiunit, relatively short-latency burst of spikes (Fig. $3 A$, lowest trace). (In this and all figures to follow, the top trace is the optic tract volley.) However,
TABLE I

Summary of location of postsynaptic units recorded in 17 successful experiments based on histological reconstruction of electrode tracks ${ }^{a}$

\begin{tabular}{ccccccr}
\hline Age & LGNv & Border & LGNd & MIN & Unknown $^{b}$ & Total \\
\hline Fetuses & & & & & & \\
E39 & 1 & & & & & 1 \\
E40 & 1 & 1 & 2 & & & 4 \\
E42a & 5 & & & & & 5 \\
E42b & & & & & 8 & 8 \\
E43a & 2 & 1 & & & & 3 \\
E43b & & 1 & 1 & & & 2 \\
E47 & & & 5 & & & 5 \\
E48 & & & & & 1 & 1 \\
E49 & & & 9 & & & 9 \\
E51 & & & 11 & & 7 & 7 \\
E52 & & & & & 7 & 7 \\
E53 & & & & & & 17 \\
E54 & 5 & 1 & 11 & & & 6 \\
E59 & & & 6 & & 23 & 86 \\
Total & 14 & 4 & 45 & & & \\
Newborns & & & & & & 14 \\
P0 & & & 12 & 2 & & 7 \\
P1 & & & 7 & & & 12 \\
P2 & & & 10 & 2 & & 33 \\
Total & & & 29 & 4 & & \\
\hline
\end{tabular}

${ }^{a}$ Six other animals were studied but results are not included here due to the poor condition of the preparation as indicated by small and inconsistent volleys that did not recover significantly.

${ }^{b}$ Probably in LGNd rather than MIN.
A

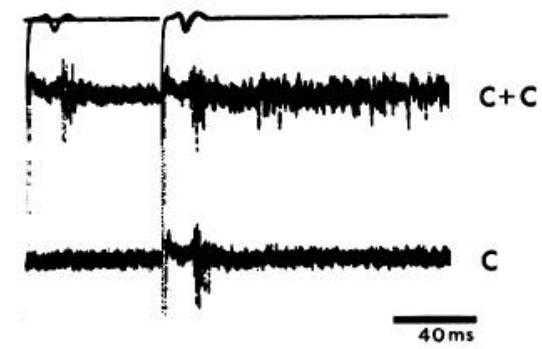

B

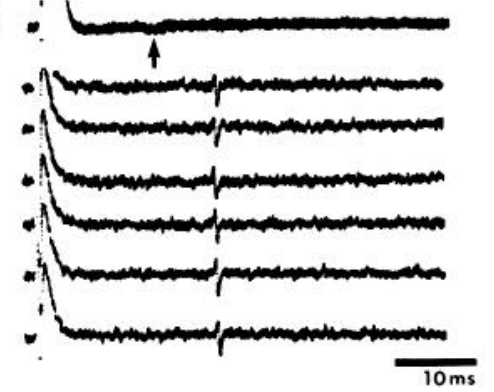

Figure 3. Examples of recordings from the LGNd of an E43 preparation. In this and all subsequent figures showing electrical recordings, the top trace is the optic tract volley. A, Lower traces: Multiunit responses elicited by stimulation of the contralateral nerve with a single maximal shock (bottom trace) or a double shock (middle trace). B, Example of a single afferent unit from a different E43 fetus. Six successive sweeps with a stimulus to the contralateral nerve are shown. (Note expanded time scale compared with $A$ and higher amplification for optic tract volley.) The threshold for the unit was very close to threshold for the volley, which is just visible on the top trace (arrow). 
following a double shock (Fig. $3 A$, middle trace), a distinctly different group of spikes appeared at much longer and more variable latencies. Because these longer-latency spikes were so variable and because they were dependent on temporal summation, we have interpreted them as representing discharges from postsynaptic LGN neurons, whereas the first short-latency burst represents discharges from the incoming afferent fibers.

We used the three criteria mentioned above to identify postsynaptic responses recorded within the LGN: long latency, large variation in latency, and either (or both) temporal or spatial summation. The identification was almost always unambiguous, being uncertain for only two units, both in neonatal animals. These units were excluded from further consideration. Several examples of successful identifications by these criteria follow.

First, an example of a single afferent unit recorded from the LGNd of an E43 fetus is shown in Figure $3 B$ to illustrate the remarkably constant latency seen on a trialby-trial basis even near threshold (as shown in the figure). Also, very little variation in latency $(0.5 \mathrm{msec})$ with variation of stimulus strength was found. Many such afferent spikes were encountered at all ages; 14 were
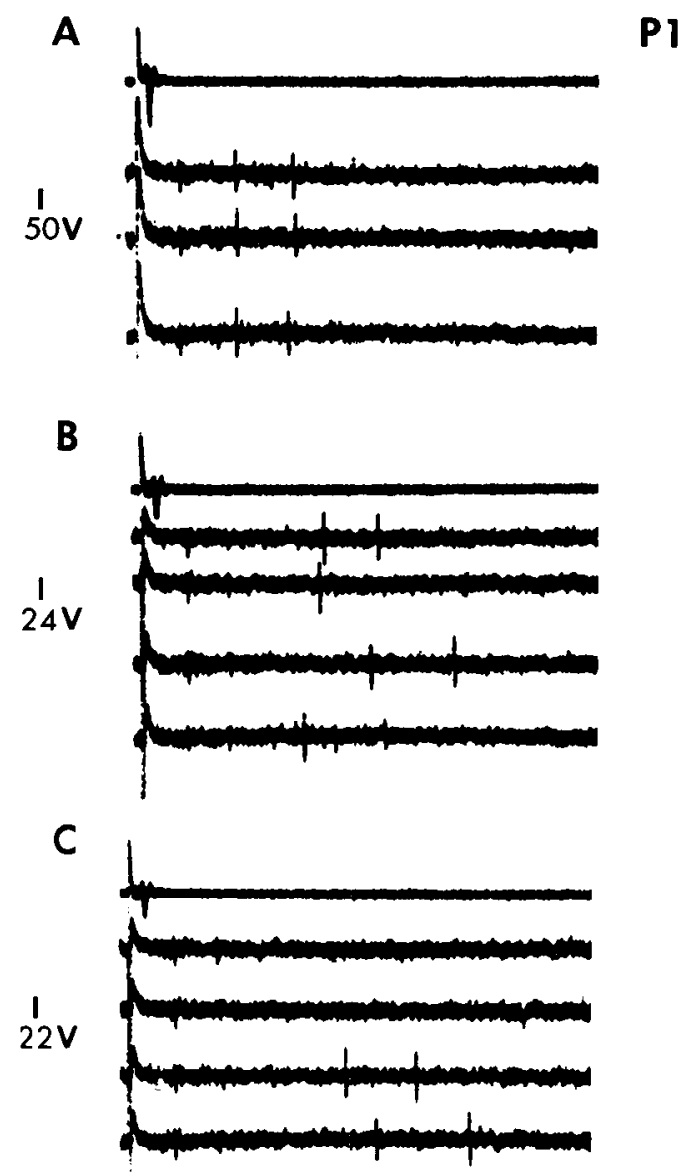

$40 \mathrm{~ms}$

Figure 4. Example of a postsynaptic single unit from a $\mathrm{P} 1$ preparation. In $A, B$, and $C$, three or four successive single sweeps are shown with stimulation of the ipsilateral nerve at the indicated voltages. Note that as the stimulus is decreased, the unit fires with increased latency and reduced probability. The optic tract volley (upper traces) also decreases. studied in detail. Latencies from stimulation to first spike ranged from 4.7 to $29 \mathrm{msec}$ without an obvious relationship to age of the fetus. (Note that both distance and conduction velocity vary with age.) The maximum variation in latency (both trial-by-trial and with variable stimulus strength) was $1 \mathrm{msec}$, except for one unit that fired at either $10 \mathrm{msec}$ or $8 \mathrm{msec}$ as stimulus strength varied but not between: this situation arose presumably due to stimulation at two different sites. In contrast, most postsynaptic units gave latency variation on a trialby-trial basis greater than $1 \mathrm{msec}$, and latency varied well beyond $1 \mathrm{msec}$ with variation in stimulus strength. The only exceptions were a few postsynaptic units in the neonatal kittens (see Fig. 11).

Next, the responses of a postsynaptic unit recorded from the LGN at P1 are shown in Figure 4. At high stimulus strengths (Fig. $4 A$ ) this unit might almost have been mistaken for spikes from a pair of afferents, although the second spike shows a latency variation of 4 msec. However, when the stimulus was turned down (Fig. $4, B$ and $C$ ), the latency became much longer and more variable, and this was related to the stimulus strength. The number of spikes per stimulus also became more variable.

The third example (Fig. 5) shows a unit identified as postsynaptic in an E53 fetus not only by means of its variable latency, but also by the dependence of its response on temporal summation. Here, a maximal shock to the ipsilateral nerve elicited only one spike, with a long (70 msec) and variable latency (Fig. $5 A$ ). However this unit could be made to fire at a short and more constant latency by employing temporal summation, i.e., double shocks (Fig. $5 B$ ). Temporal summation was also shown for this unit by turning the stimulus down below threshold for firing with a single shock (Fig. $5 \mathrm{C}$ : note smaller tract volley) and then giving a double shock to the ipsilateral nerve at this intensity, thereby eliciting shorter-latency spikes (Fig. $5 D$ ). It should be noted that the summation seen here could include presynaptic facilitation (del Castillo and Katz, 1954).

A fourth example (Fig. 6) is of a postsynaptic unit at E54 which satisfied the criteria above but also possessed an additional postsynaptic property: that of firing a burst of spikes to each single shock. We consider it highly unlikely that this was a multiunit recording because the different spike components in the burst, although recruited in sequence with stimulus strength, always appeared in the same sequence and varied in latency in a stereotyped fashion when stimulus strength was varied. (An example of a burst of spikes which, in contrast, did not clearly act as one unit may be seen in Fig. 11, where the late, small spike has a variable time relationship with the second of the large spikes.) The complex shapes of spikes shown in Figure 6, with an apparent decomposition of the spike into separate components as the burst (and presumably the underlying depolarization) progresses, was common to most units firing in bursts. We do not know whether this represents a change in spike shape at one location, a decoupling of the spike mechanism in different parts of the postsynaptic cell, or possibly even electrical coupling between LGN neurons (cf. Fulton et al., 1980). 

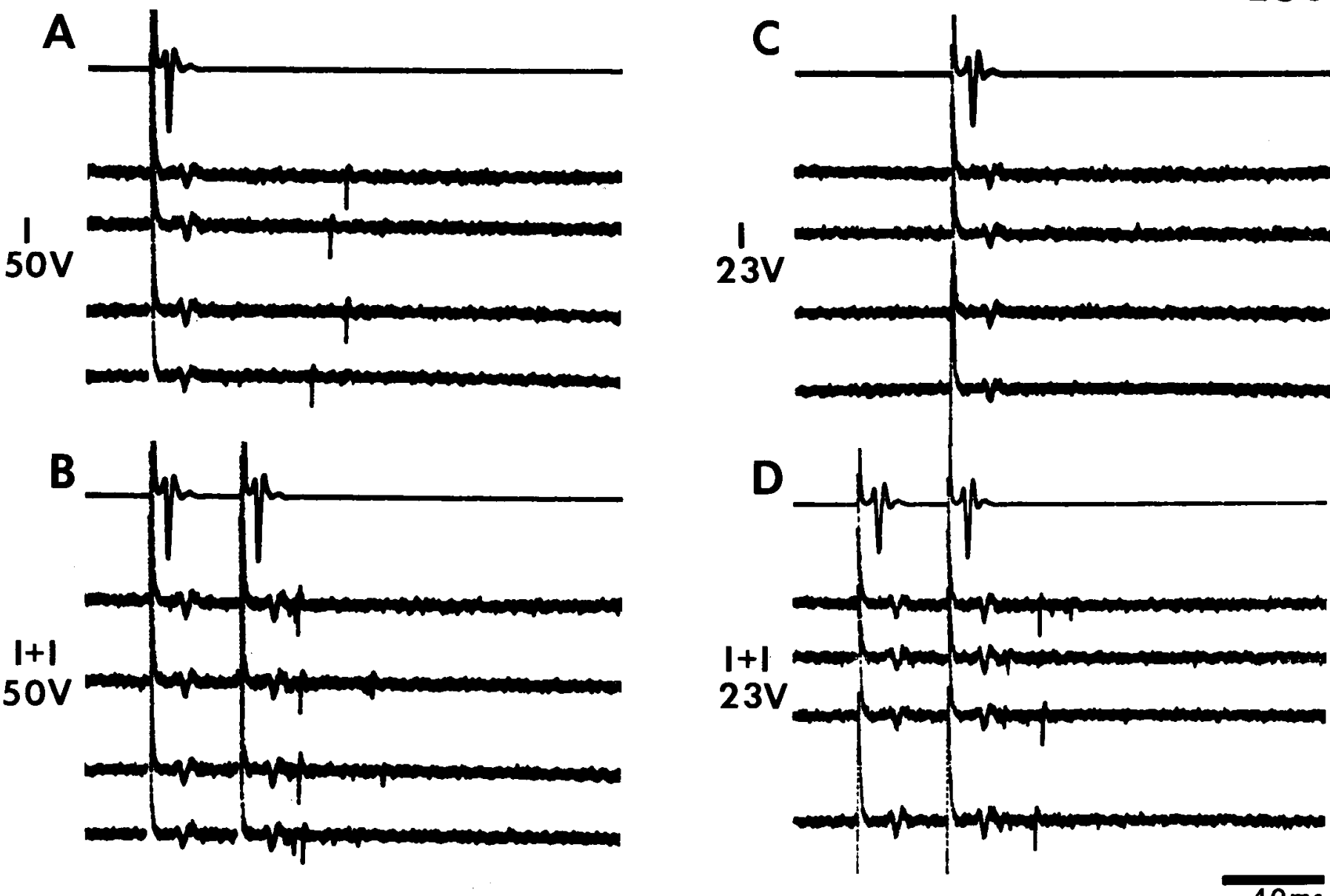

$40 \mathrm{~ms}$

Figure 5. Demonstration of temporal summation for a unit from an E53 preparation. In $A$ to $D$, four successive single sweeps are shown with stimulation of the ipsilateral nerve at the indicated voltages. $A$ and $C$, Single shocks; $B$ and $D$, double shocks. Note the shorter latency and higher probability of firing with double shocks.

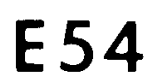

As described above, single-unit postsynaptic responses often habituated, were very variable, and were frequently recorded in the presence of other units. These characteristics made it difficult to assess convergent effects from the two nerves, to measure variation in response with stimulus strength, and to estimate the time courses of excitation and inhibition. To overcome these problems we used certain standard procedures that are illustrated by the final example. Figure 7 shows recordings from a unit in an E51 fetus that received convergent excitation from each nerve stimulated separately (Fig. 7, $A$ and $B$ ). To ensure that the same unit was excited from both nerves, the standard procedure was to turn down both stimuli to just below threshold and then to give them both together, either simultaneously or at an appropriate latency. This procedure is illustrated in Figure $7 \mathrm{C}$, in which the sequence of stimulation was chosen specifically to avoid habituation effects (see Fig. 7 legend). Accordingly, Figure $7 C$ (third trace) shows that stimuli which were subthreshold when delivered to either nerve alone

Figure 6. Example of a unit in an E54 preparation that fired a burst of spikes to each stimulus. Three successive single sweeps with a maximal stimulus to the ipsilateral nerve are shown. evoked clear single-unit spikes when delivered together with the appropriate timing, thereby proving that this LGN unit receives convergent excitation from both nerves.

The procedure outlined above requires the use of a minimal stimulus, thereby also minimizing the risk of 
E51

A

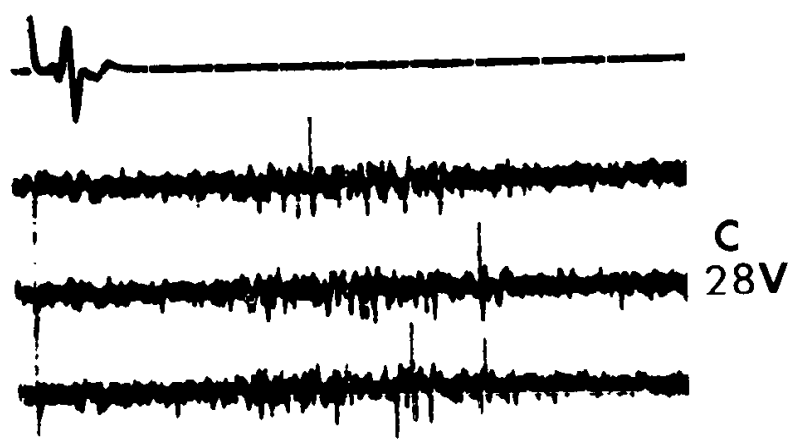

B

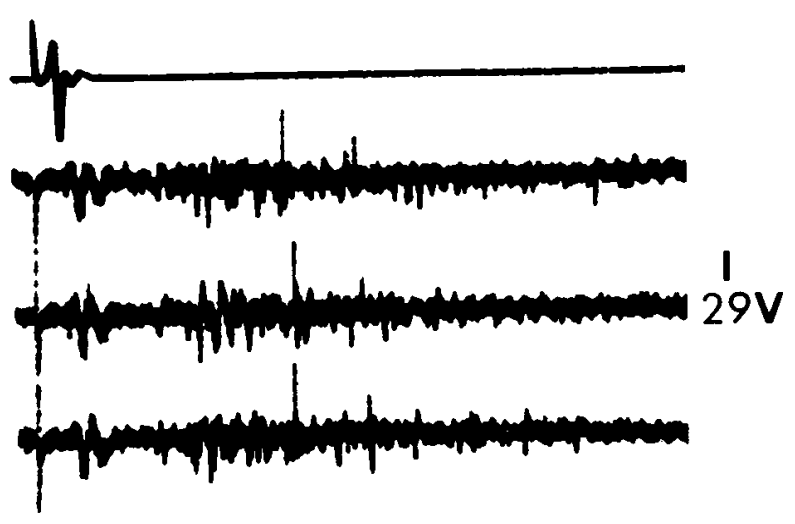

C

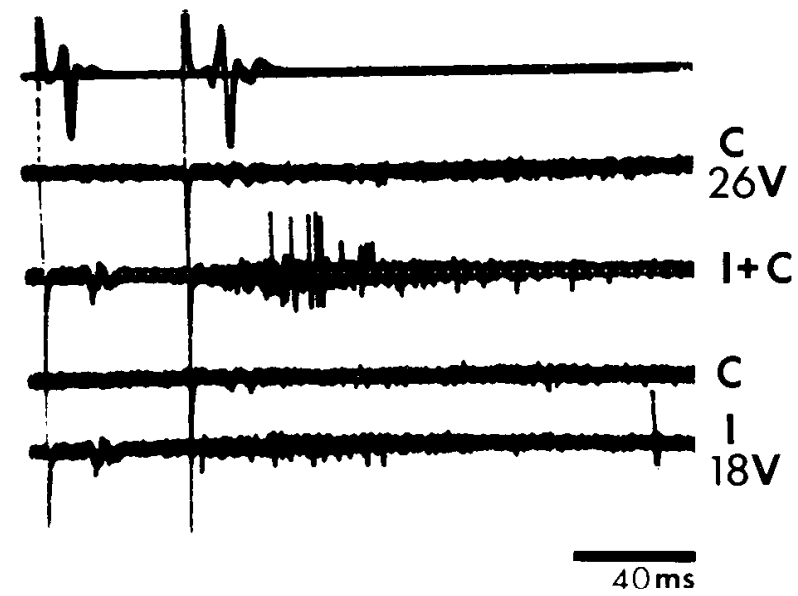

Figure 7. Convergent excitation from the two nerves onto a single unit in an E51 preparation. $A$ and $B$, Three successive single sweeps with moderate stimuli delivered to the contralateral $(A)$ or ipsilateral $(B)$ nerve. In each sweep a large positivegoing single-unit spike stands out. $C$, To prove that the same unit is evoked by the two separate stimuli a standard stimulation sequence is used. Stimulus strength is reduced for each nerve, as indicated. Traces 2 to 5 each have five sweeps superimposed. Responses were recorded in the following sequence at a regular rate of $0.2 \mathrm{sec}^{-1}$ : First a test stimulus to the contralateral nerve is turned on but the recording is not begun until the response is habituated. Then (second trace), five sweeps are superimposed: no spikes are evoked. Next (third trace), five stimuli are preceded $(45 \mathrm{msec})$ by a conditioning (ipsilateral) stimulus and are superimposed: each stimulus evokes a large single unit spike. In the fourth trace, the next five test stimuli are repeated alone as a control for variability with time. Finally, as another control in the fifth trace the test stimulus is turned stimulus spread. (In that case, however, no stimulus spread was suspected-see Fig. 1, $A$ and $B$ ). Moreover, the procedure also sometimes revealed units receiving convergent excitation not noticed previously (e.g., the unit represented by the small positive-going spikes in Fig. $7 C$ ), and it was the only way of demonstrating convergent excitation in some units that did not respond to both nerves stimulated separately. We regard responses assessed by this procedure to be unequivocal evidence for convergent excitation (even if polysynaptic) from both nerves onto single units in the LGN.

Postsynaptic responses recorded from neonatal preparations. By birth, retinal afferents from the two eyes have nearly completely segregated from each other, and the adult-like laminar pattern of input is present within the LGN (Shatz, 1983). Some characteristic features of adult organization can be found at the ultrastructural level as well, the most notable being the fundamental arrangement of retinal terminals as central presynaptic elements in synaptic glomeruli (Guillery, 1969; Mason, 1982; C. J. Shatz and M. W. Siegel, manuscript in preparation). Thus, although much further refinement of connectivity continues during the immediate postnatal period (Kalil and Scott, 1979; Winfield and Powell, 1980; Mason, 1982), we considered it likely that some of the basic adult circuitry was present at birth. Therefore, we began our investigation by making extracellular microelectrode recordings from the neonatal LGN preparations in our chamber in order to obtain some verification that the in vitro techniques were reliable before we applied them to the study of prenatal development of the retinogeniculate pathway.

In the adult LGN, electrical stimulation of the optic nerves commonly elicits a mixture of excitation and inhibition in postsynaptic neurons (Lindström, 1982; see Godfraind and Kelly, 1981, or Jones, 1981, for earlier references). For example, as well as receiving powerful monosynaptic excitation from one optic nerve, neurons situated in the lamina innervated by that nerve also receive disynaptic (and trisynaptic) inhibition from both that nerve and the other. We therefore hoped to find examples of such synaptic physiology in our recordings from neonatal preparations.

Extracellular recordings from a single unit that could be inhibited by optic nerve stimulation at postnatal day 2 are shown in Figure 8 . Each single shock to the ipsilateral nerve evoked a relatively stereotyped pattern of spikes. However, when this stimulus was accompanied simultaneously by a maximal stimulus to the contralateral nerve, the excitatory response was totally abolished. Stimulation of the contralateral nerve alone had no effect (not shown).

The synaptic physiology of this single unit indicated that it was likely to be located within an LGN lamina receiving afferent input from the ipsilateral retina. This suggestion was verified by the histological reconstruction of the electrode track, as shown in Figure 9. At the top is a cresyl violet-stained section that contains a lesion

off and five sweeps are superimposed with the conditioning stimulus alone. No time for habituation is given here, in order to match the conditions in trace 3 . 


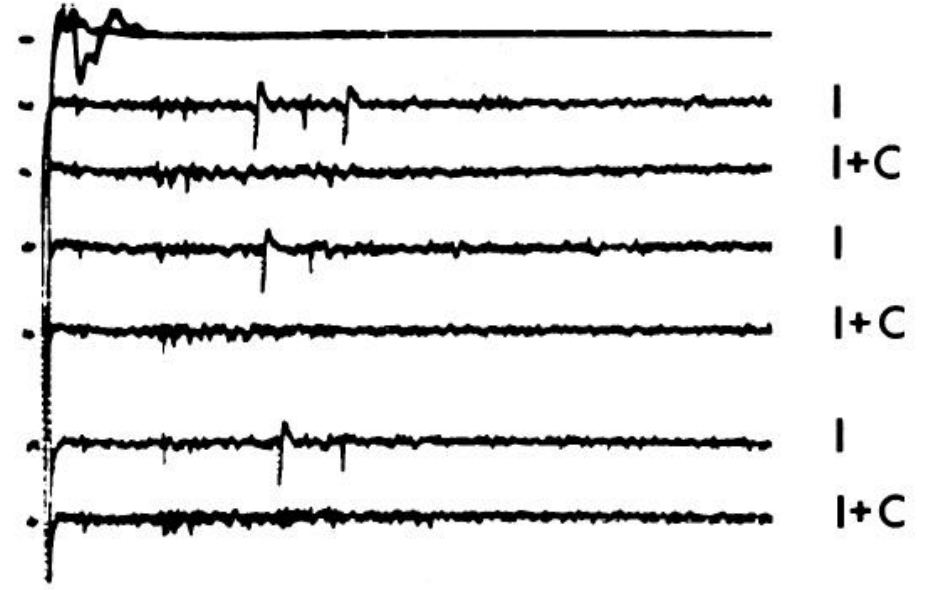

$20 \mathrm{~ms}$

Figure 8. Inhibition of a unit in a P2 preparation. Six successive single sweeps are shown; the second, fourth, and sixth traces $(I)$ are the single-unit response to the ipsilateral stimulus $(60 \mathrm{~V})$ alone. The third, fifth, and seventh traces $(I+C)$ show inhibition when ipsilateral $(60 \mathrm{~V})$ and contralateral (100 V) stimuli are given simultaneously. No response was observed to the contralateral stimulus alone (not shown). This unit was recorded at site 11 in Figure 9.

(Fig. 9A, arrow) made very near the unit, toward the end of the track. The animal used here had previously received an injection of $\left[{ }^{3} \mathrm{H}\right]$ leucine into the contralateral eye. Thus, the autoradiograph of Figure $9 B$ shows, in an adjacent section, the relationship between contralaterally (labeled) and ipsilaterally (unlabeled) innervated laminae and the reconstructed electrode track (Fig. $9 B$, white line). The unit illustrated in Figure 8 is unit 11 here - clearly located within ipsilaterally innervated lamina $\mathrm{A} 1$, as expected. Furthermore, nearby unit 13 received a similar mixture of excitation from the ipsilateral nerve and inhibition from the contralateral nerve, and was also located within lamina A1. Recordings from this unit are shown in Figure 10, in which the time course of inhibition was also examined by varying the interval between stimulation of the contralateral and ipsilateral nerves. Measurements of the latency to the first spike (Fig. 10B) indicate that inhibition became maximal by roughly 30 $\mathrm{msec}$ and was quite long lasting $(350 \mathrm{msec})$. This particular time course was common to every age at which inhibition could be recorded in vitro and bears reassuringly close resemblance to that found in vivo in the adult cat's LGN (Suzuki and Kato, 1966; Kato et al., 1971; Ono and Noel, 1973).

Unit 13 demonstrated another response property of at least some cells in the adult LGN, namely the ability to fire in an all-or-none fashion as stimulus strength is varied. This is thought to be a reflection of the fact that single afferent EPSPs are known to bring some LGN neurons to threshold (e.g., Cleland et al., 1971). Such behavior is illustrated in Figure 11. At and below a stimulus stength of $69 \mathrm{~V}$ to the ipsilateral nerve, no response was visible. At $70 \mathrm{~V}$ and above, a stereotyped burst of spikes was present almost independent of stimulus strength. This burst may represent one or several units (which could all be excited by a single afferent). It is tempting to believe that the small spike at the start of the burst (Fig. 11A, arrow), which has the same threshold, represents the afferent concerned. All of the later spikes, but not this one, were definitely postsynaptic since they were inhibited by a stimulus to the contralateral nerve (see Fig. 10A). Two additional units, one each recorded in the $\mathrm{P} 0$ and $\mathrm{P} 1$ preparations had similar very sharp thresholds.

Single units receiving similar mixtures of excitation and inhibition from stimulation of both nerves were found in all three of the neonatal animals studied here. (Both excitation and inhibition were recorded from 7 of 11 LGNd units at P0, 6 of 6 units at P1, and 6 of 10 units at P2.) These results were especially encouraging since they indicated that at least one aspect of the intrinsic circuitry of the LGN-inhibition-had survived the surgical manipulations and was functioning in the in vitro preparations.

However, not all cells in the adult LGNd receive excitation from one eye only and inhibition from the other.
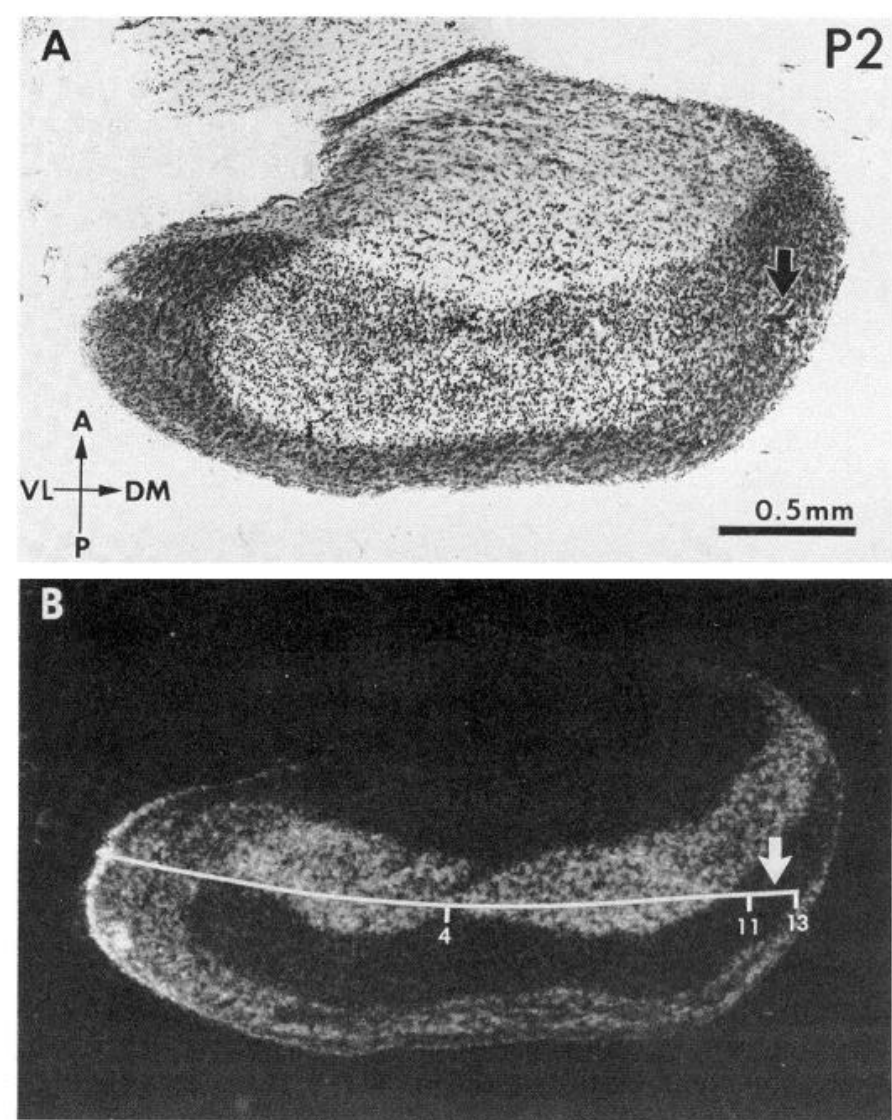

Figure 9. Reconstruction of an electrode track in a P2 preparation. A, A cresyl violet-stained section showing LGN with electrode entry point (damaged tissue on left) and a lesion near the end of the track (arrow). Cellular lamination can just be discerned, although it is somewhat obscured by the necrotic region. $B$, Darkfield autoradiograph prepared from an adjacent section, with electrode track drawn in. The contralateral eye had been injectd with $\left[{ }^{3} \mathrm{H}\right]$ leucine $24 \mathrm{hr}$ before the in vitro experiments. Regions containing radioactive label appear white. Recording site 11, location of unit in Figure 8; site 13, location of unit in Figures 10 and 11; site 4, see the text. Arrow, site of lesion. 


\section{P2}
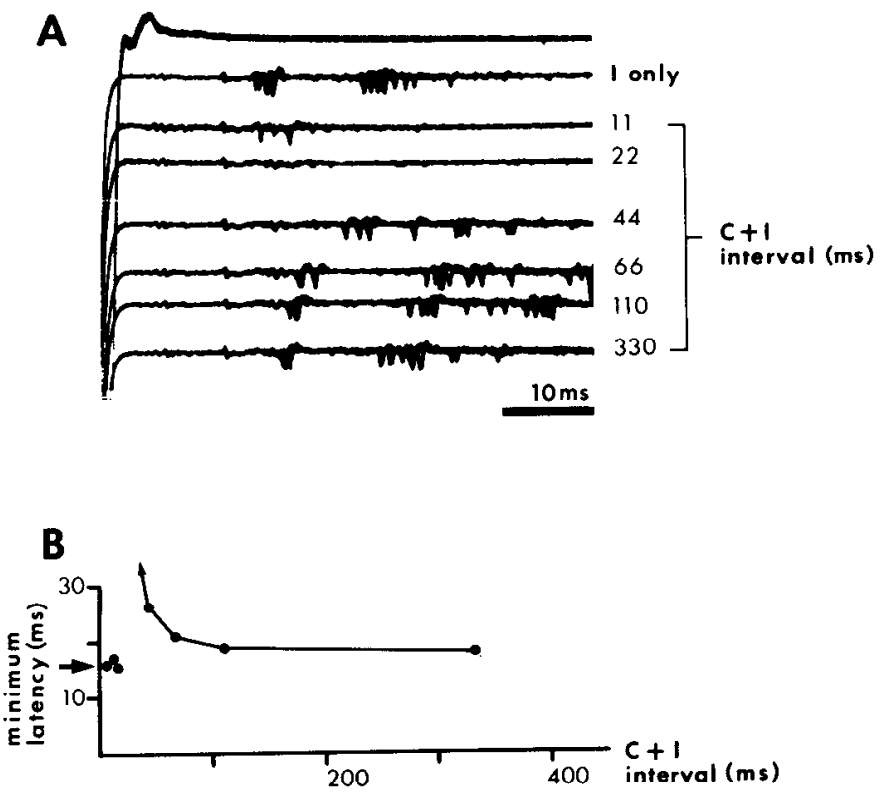

Figure 10. Measurement of the time course of inhibition for unit 13 in Figure 9 (P2 preparation). A, Each of the lower seven traces has four sweeps superimposed. The start of the sweeps is the time of the (test) ipsilateral stimulus. Second trace, Ipsilateral stimulus ( $80 \mathrm{~V}$ ) alone. Remaining six traces, Ipsilateral stimulus preceded by (conditioning) contralateral stimulus $(100 \mathrm{~V})$ at the indicated intervals. Note increased latency of spikes, even at 330 -msec interval. No response was observed to the contralateral stimulus alone (not shown). $B$, Graph of latency to first spike versus conditioning-testing interval. The arrow indicates minimum latency to test stimulus alone.

A small proportion receives excitation, even if weak, from both (Sanderson et al., 1971). These units are commonly located near or within the interlaminar zones. Therefore, it was not surprising to find in the neonatal preparations units near the laminar borders that, when tested as described in the previous section, displayed convergent excitation. One such unit was found at recording site 4 of Figure $9 B$. We found clear-cut evidence for convergent excitation in 11 of 21 units tested in the two neonatal preparations ( $\mathrm{P} 0$ and $\mathrm{P} 2$ ) in which no stimulus spread could be detected, even up to maximal stimulus strengths (see "Materials and Methods" and Table II, column C). As compared with the adult in which units with such properties are rare, this is a remarkably large proportion $(52 \%)$ of the entire sample. It is perhaps not so surprising, however, in view of the fact that in the neonatal animals afferents from the two eyes still overlap each other slightly at the laminar borders (Shatz, 1983, especially Fig. 13), where the majority of our microelectrode recordings were located at these ages (see Figs. 9 and 14).

In the neonatal preparations we also recorded from several units which received a more complex mixture of excitation and inhibition than that described above. For instance, 4 of 21 units tested in the LGNd at P0 and P2 received convergent excitation from both nerves and also inhibition from one nerve (see Table II, column D). Recordings from such a unit are shown in Figure 12. Here, stimulation of the ipsilateral nerve alone elicited spikes in 5 of 5 trials (Fig. 12A). The spikes could be abolished by stimulation of the contralateral nerve 10 msec before ipsilateral nerve stimulation (Fig. 12B), indicating that this unit received a mixture of excitatory input from the ipsilateral nerve and inhibitory input from the contralateral nerve. In addition, the unit received a very early and short-duration excitatory input from the contralateral nerve as shown in Figure 12C. Here, timing of the two stimuli was adjusted so that the ipsilateral shock preceded the contralateral shock by 2 msec. The resulting three spikes (Fig. 12C, third trace) were of shorter latency than any evoked to stimulation of the ipsilateral nerve alone (Fig. 12C, second and fourth traces), indicating that the contralateral nerve supplies additional subthreshold excitation to this unit.

Another group of units received excitation from only one nerve but convergent inhibition from both. A recording from one such unit, also at P0, is shown in Figure 13. Stimulation of the ipsilateral nerve at increasing stimulus strengths first excited the unit at progressively shorter latencies (Fig. 13A: 20 to $27 \mathrm{~V}$ ), but then completely abolished the response (Fig. 13A: $33 \mathrm{~V}$ ). Thus, this unit receives both excitatory and inhibitory influences from the ipsilateral nerve, the net mixture depending on stimulus strength. The unit was also inhibited by stimulation of the contralateral nerve, as shown in Figure $13 B$.

To determine whether there was any systematic relationship between the location of these units within LGN laminae at $\mathrm{P} 0$ and their more complex responses to optic nerve stimulation, the electrode track containing these and units recorded at 14 other sites was reconstructed and is shown in Figure 14. Here again, as in Figure 9, the track traversed the LGN from lateral to medial. The first four units recorded (all close to lesion 1) were within the lamina $\mathrm{Al}$ and all received excitation from stimulation of the ipsilateral nerve. The electrode next traveled within lamina A close to the border with lamina A1, but did not record from any postsynaptic units receiving
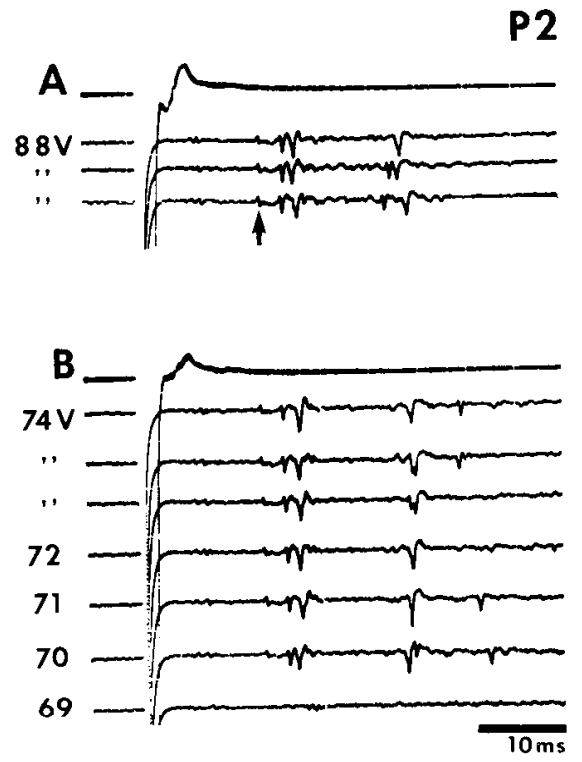

Figure 11. Example of a postsynaptic unit at P2 with a sharp threshold (same unit as in Fig. 10). A, Three single successive sweeps with a constant suprathreshold stimulus $(88 \mathrm{~V})$ to the ipsilateral nerve. $B$, Successive single sweeps at the indicated voltages. Note that the small initial spike (arrow in $A$ ) has the same threshold as the later spikes (see the text and Fig. 10). 
PO
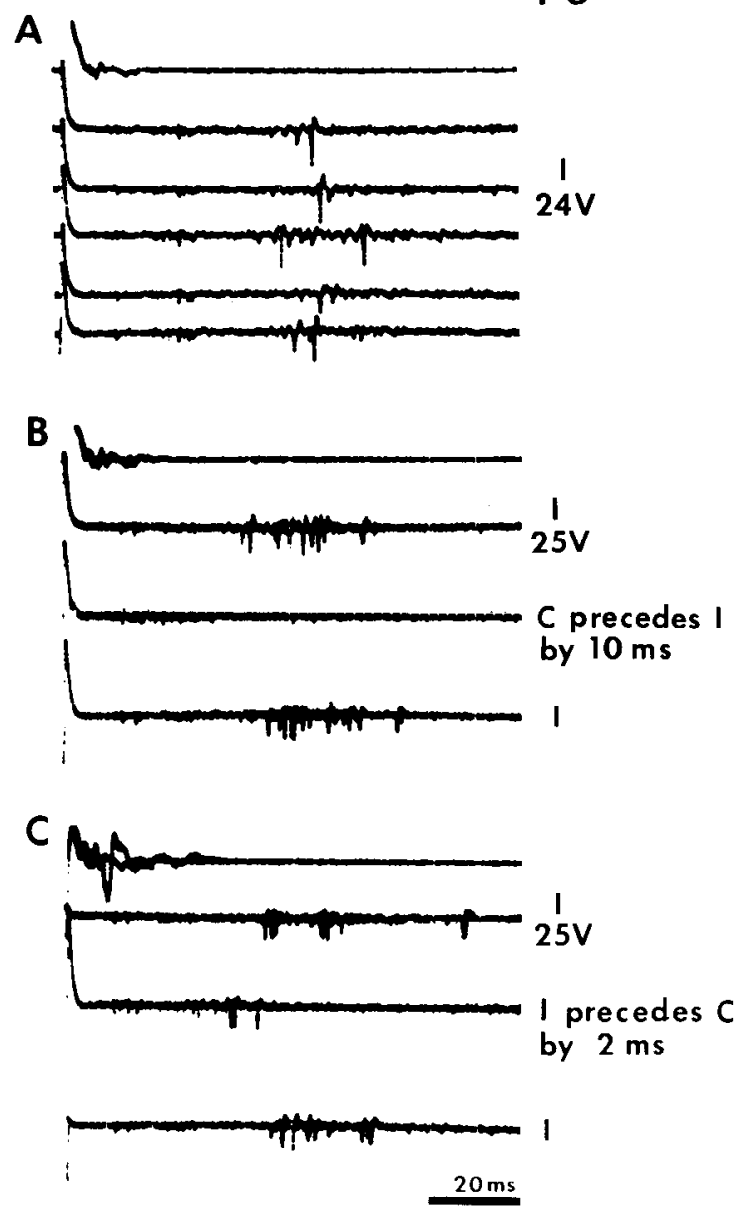

Figure 12. Responses of a unit from a P0 preparation that was excited from both nerves and inhibited from one of them. $A$, Four successive single sweeps with a stimulus of $24 \mathrm{~V}$ to the ipsilateral nerve to show variability of the response. $B$ and $C$, Constant $(25 \mathrm{~V})$ stimulus to the ipsilateral nerve with (third trace) and without (second and fourth traces) conditioning stimuli $(27 \mathrm{~V})$ to the contralateral nerve at indicated intervals. Three sweeps are superimposed for each trace (sweeps start at the time of the ipsilateral stimulus for $B$ and at the time of the contralateral stimulus for $C$ ). Note that conditioned responses in $C$ (lhird trace, 2-msec interval) have a shorter latency than any of the others. The tract volleys were checked for stimulus spread ( $80 \mathrm{~V}$ to each nerve) at the time of this recording, and none was observed. This is unit 4 in Figure 14.

excitation from the contralateral nerve (unit 6 was an afferent) until well within lamina A (units 9 to 11). Most of the units receiving a more complex mixture of excitation and inhibition were located near laminar borders, including units illustrated previously in Figures 12 (unit 4) and 13 (unit 5).

The results of all recordings from the two neonatal preparations in which stimulus spread was definitely not present are summarized in Table II according to unit response properties. Of the 21 units studied thoroughly, 10 received unilateral excitation from one nerve only: one was exclusively monocular (Table II, column A) and the remaining nine received unilateral excitation plus inhibition from one or both nerves (Table II, column B). Of these nine units, six received a simple mixture of excitation from one nerve and inhibition from the other, and the other three received an even more adult-like mixture of excitation from one nerve and inhibition from both (Jones, 1981; Lindström, 1982). In contrast, 11 of the 21 units received bilateral excitation from the two nerves: 7 of these received simple dual excitation from both nerves (Table II, column C), whereas 4 received an additional inhibitory input from one nerve only (Table II, column D). Despite the small sample size here, these results differ profoundly from those described immediately below for units recorded in the fetal LGN preparations.

Postsynaptic responses recorded from fetal preparations. The earliest age studied was E39. In this preparation, one definitely postsynaptic unit was recorded in the LGNv (see Table I). At E40, four postsynaptic units were recorded, and reconstruction of the electrode track revealed that two of these were located in the LGNd. Figure 15 shows microelectrode recordings from one of these units. A single maximal shock to the contralateral nerve elicited a multiunit burst, most likely afferent discharges,
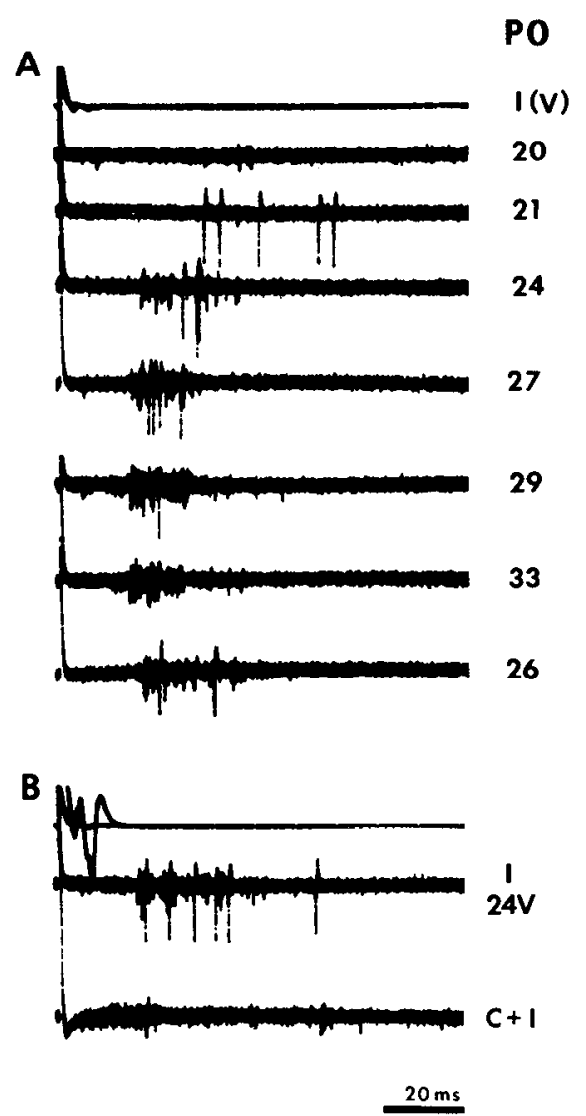

Figure 13. Reponses of a unit from a P0 preparation which was excited from one nerve and inhibited from both nerves. $A$, Responses with increasing stimulus strength to the ipsilateral nerve (voltages as indicated). Five sweeps are superimposed for each trace, with time allowed for habituation after each change of stimulus. The unit only fired between $21 \mathrm{~V}$ and $29 \mathrm{~V}$. (The volley had a threshold of $19 \mathrm{~V}$ and had a maximum of $80 \mathrm{~V}$ ). $B$, Response of the unit to the ipsilateral nerve at the "best" voltage $(24 \mathrm{~V})$ with and without simultaneous stimulation of the contralateral nerve $(80 \mathrm{~V})$. Five sweeps are superimposed for each trace. This is unit 5 in Figure 14. 

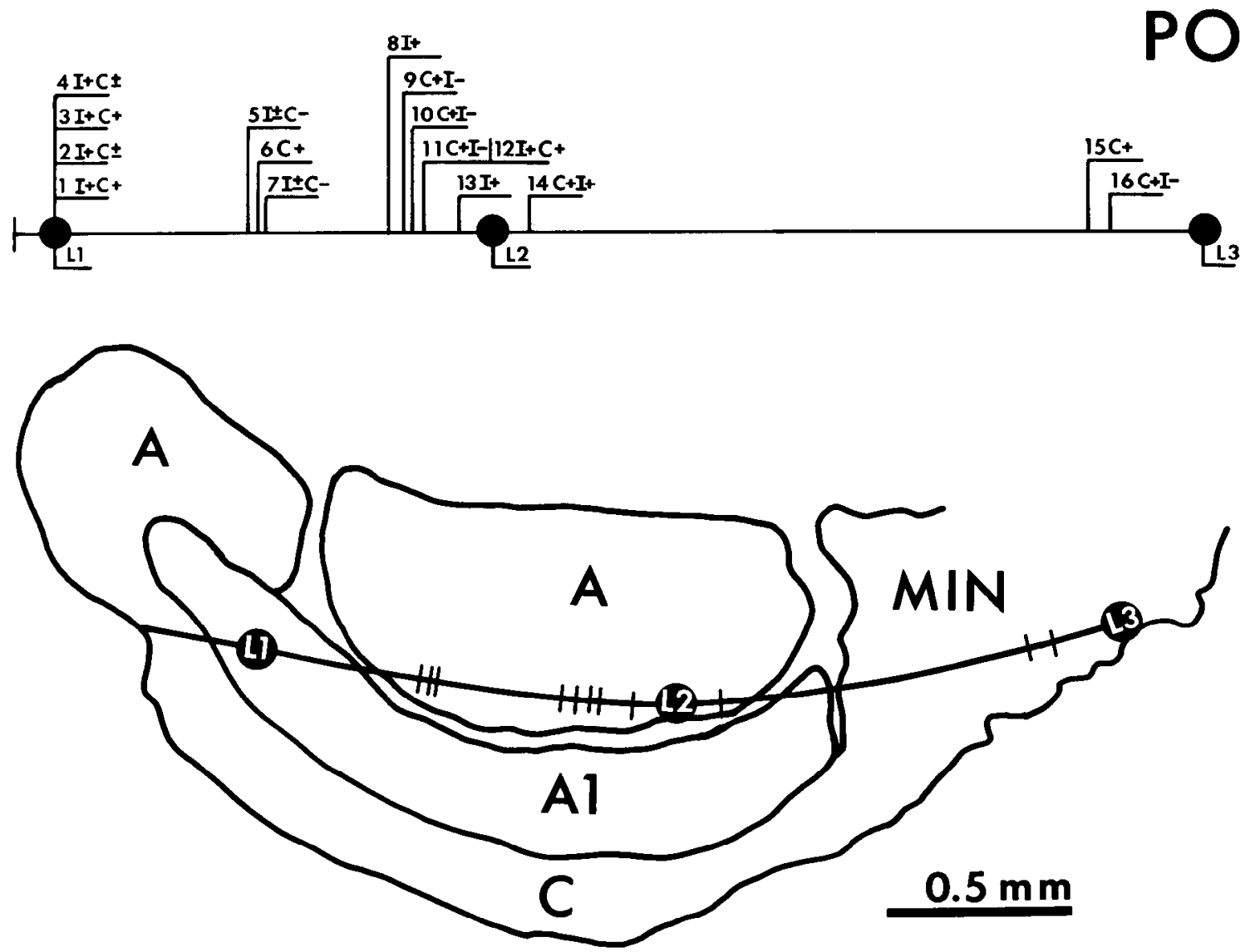

Figure 14. Reconstruction of an electrode track in the P0 preparation (camera lucida tracing from cresyl violet-stained sections). In this plane of section, anterior is up, posterior is down, ventrolateral is to left, and dorsomedial is to right, as in Figure 9. Three lesions were made to define the track ( $L 1$ to $L 3$ ). Marks along the track indicate recording sites, and the corresponding response properties of the units are shown above, where the position along the track is scaled according to the electrode microdrive reading. During the early part of the track, some swelling of the preparation occurred, accounting for the successive recording of four different units, apparently at the same site. During the latter part of the track some dimpling occurred, accounting for the apparently greater distance between $L 2$ and $L 3$ than is indicated in the histological drawing. $I \pm C+$ indicates a cell excited by both nerves and inhibited by the ipsilateral nerve, and so on. Unit 6 was an afferent, unit 13 was not tested with a contralateral stimulus, and site 14 was a multiunit recording. All other sites represent single postsynaptic units fully tested from both nerves.

E40

A

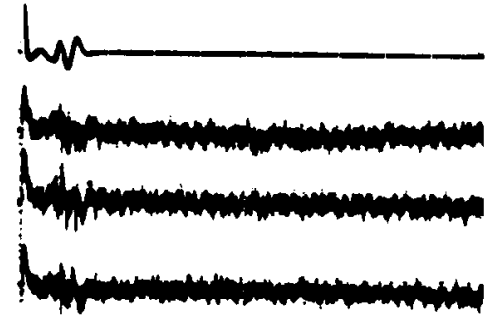

B

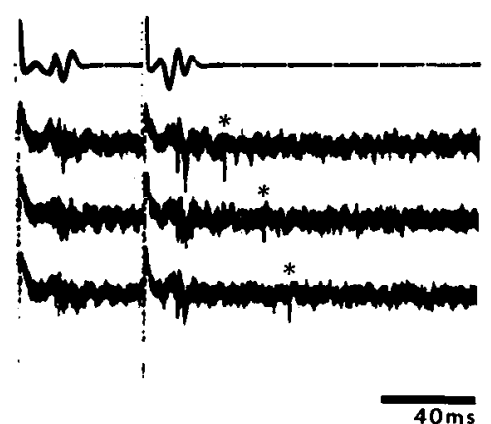

at a relatively short latency (Fig. 15A). In contrast, a double shock evoked an additional single unit with a very variable latency (Fig. 15B, asterisk). In view of the variability in latency and dependency on temporal summation, it is highly likely that this unit is postsynaptic.

It is worth noting, here, that the volley recorded from the optic tract was itself potentiated with double shocks (Fig. 15B, top trace), as was the case in several of the youngest fetuses. Here the potentiation is unlikely to be due to recruitment of additional fibers, since the stimulus was already maximal. A more likely cause is extracellular $\mathrm{K}^{+}$accumulation, as observed in neonatal rat optic nerve (Connors et al., 1982). Potassium would act to depolarize afferent fibers and increase their conduction velocities, thereby altering synchronization within the volley (note the change in volley shape and latency in Fig. 15B).

Figure 15. A postsynaptic unit (spikes marked by asterisk) recorded in LGNd of an E40 preparation. Three successive single sweeps with single $(A)$ and double $(B)$ maximal shocks delivered to the contralateral nerve. 
E43

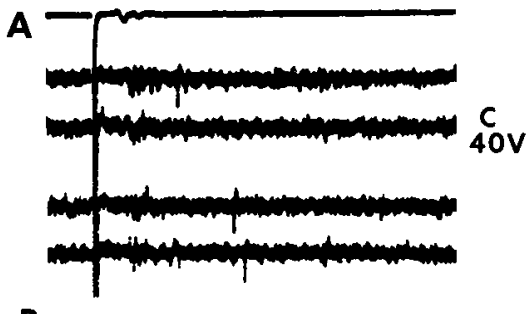

B

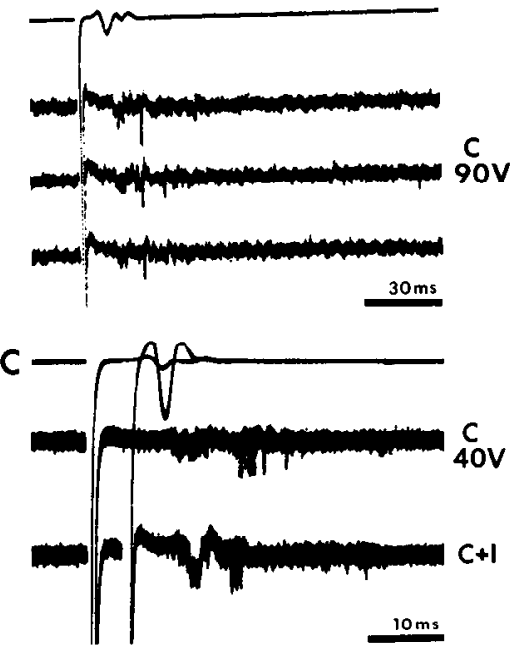

Figure 16. Weak convergent excitation seen in a unit in an E43 preparation. $A$ and $B$, Successive single sweeps at two different stimulus strengths to the contralateral nerve to show variability of response and variation with stimulus strength. $C$, Eleven sweeps superimposed of stimuli to the contralateral nerve $(C, 40 \mathrm{~V})$ alone and succeeded $(4.5 \mathrm{msec})$ by stimulation of the ipsilateral nerve $(C+I$, both $40 \mathrm{~V})$. Note the shorter and less variable latency for the combined stimuli. No response was present for a stimulus to the ipsilateral nerve alone. The tract volleys were checked for stimulus spread (contralateral, $40 \mathrm{~V}$; ipsilateral, $60 \mathrm{~V}$ ) at the time of this recording, and none was present (see Fig. $1 C$ ).

Alternatively, these unmyelinated fibers could have large hyperpolarizing afterpotentials and hence large spike amplitudes to the second stimulus. Depending on which of these mechanisms was operating at the terminals, the synaptic effectiveness of the volley could be either increased or decreased independently of temporal summation (cf. Singer and Lux, 1973).

The youngest age at which convergence of inputs from the two nerves was examined was E43. (This preparation was shown to be free from the effects of stimulus spread as shown in Fig. 1C.) At this age, weak convergent excitation from both nerves onto single LGNd neurons was detected (see Table II). As shown in Figure 16, stimulation of both nerves together evoked postsynaptic spikes of shorter and less variable latency than those produced by contralateral stimulation alone at the same stimulus strength (Fig. $16 \mathrm{C}$ ). Thus, even prior to the onset of segregation at E47, functional connections can be demonstrated between retinal afferents and LGN neurons.

At older fetal ages, postsynaptic activity was more readily evoked, and it was significant to find that the majority of units studied received excitation from both nerves. In several instances, it was also possible to study the time course of excitation in some detail. Figure 17 shows such a study for a unit recorded from an E49 preparation: Figure $17, A$ and $B$, contains a series of recordings (each 5 sweeps superimposed) in which the interval between stimulation of the two nerves was varied systematically. Here, as the interstimulus interval increased up to about $100 \mathrm{msec}$, the latency measured from the ipsilateral shock (Fig. 17, $A$ and $B$ : artifacts) to the first spike decreased progressively. Figure $17 \mathrm{C}$ shows this decrease graphically and illustrates the broad period of excitation lasting roughly $300 \mathrm{msec}$. Although the latency
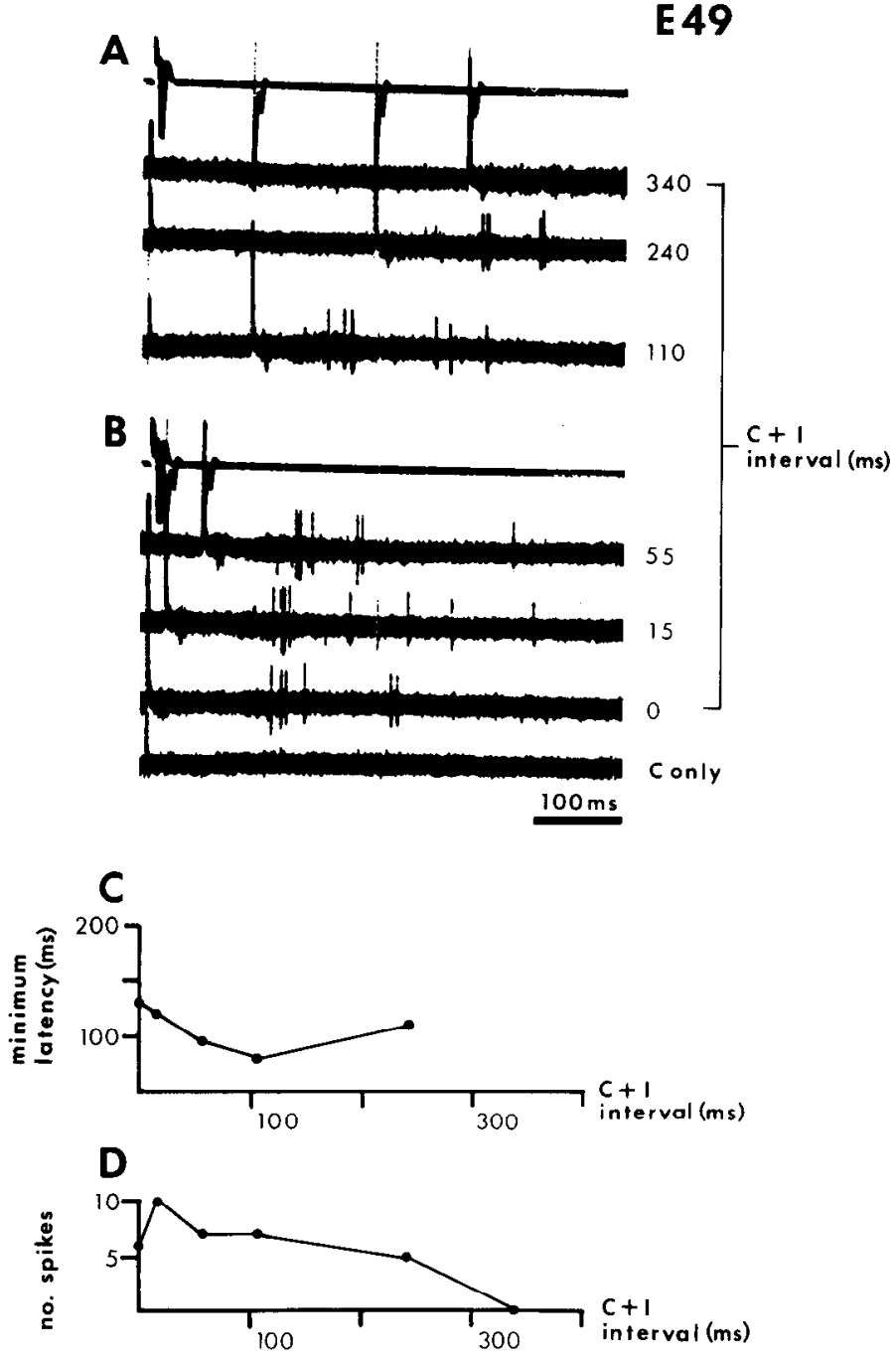

Figure 17. Time course of excitation in an E49 preparation. The time course of the response of a single unit to a (conditioning) contralateral stimulus $(80 \mathrm{~V})$ was studied with an ipsilateral nerve stimulus $(100 \mathrm{~V})$ as the test. $A$, and $B$, Five sweeps superimposed for all traces, which start at the time of the contralateral stimulus. Ipsilateral stimuli are given at the indicated times. Ipsilateral stimulus alone gave no response (not shown). C, Latency of earliest spike measured from the ipsilateral stimulus in the above records and plotted against stimulus interval. $D$, Number of spikes evoked per five stimuli from the above records plotted against stimulus interval. 
is rather long here, the overall time course of excitation revealed by this graph is typical of the fetal preparations.

Another approach was to measure the number of spikes evoked in five stimuli (Fig. 17D). This measure gives an excitability maximum at 15 msec. Neither latency nor number of spikes gives a totally accurate estimate of the time course of the underlying excitation produced by the conditioning (contralateral) stimulus, since the response to the test stimulus also has a long time course (and presumably a slow rise time). Most units were formally tested with only one or two conditioning/testing intervals. Nevertheless, it was clear that the most effective intervals were usually between 20 and $50 \mathrm{msec}$. In view of these considerations, the rise times of the synaptic responses may be deduced to be between 20 and 100 msec.

In an experiment performed at E51, in which stimulus spread was undetectable throughout the experiment (see Fig. 1), it was possible to examine systematically the relationship between single-unit response properties and position within the LGNd. All of the 10 postsynaptic single units tested were found to receive convergent excitation from the two nerves (Table II). Figures 18 and 19 illustrate the results from one of the two penetrations made in this preparation. As shown in Figure 18A, a cresyl violet stained section, the electrode traversed the LGNd from lateral to medial, stopping finally at the site marked by a lesion (Fig. 18A, arrow). By superimposing the reconstructed electrode track onto an adjacent section in which the autoradiographic labeling pattern produced by injecting the contralateral eye (at E50) is shown, it can be seen in Figure $18 B$ that the electrode traveled first through the optic tract and future C-layers (labeled) toward and into the region of future lamina A1 (unlabeled).

Since the electrode track was located within regions of the LGNd receiving input at least from the contralateral eye (Fig. 18B), we expected the majority of units recorded (Fig. 18B, black slashes) to receive excitation from stimulation of the contralateral nerve. This expectation was borne out, as shown in Figure 19, which illustrates the response properties of each unit recorded along the electrode track. Moreover, we found that each unit tested received excitatory inputs from the ipsilateral nerve as well.

The first site where postsynaptic multiunit responses could be recorded (Fig. 19, $M$ ) corresponded to the histological border between the optic tract and the LGN. Unit 1 (Fig. 19,1) was the first single unit isolated along the track. Stimulation of the ipsilateral nerve at progressively higher stimulus strengths considerably shortened the latency to first spike from this unit, confirming its postsynaptic identity. Unfortunately, the response to the contralateral nerve could not be tested because of the presence of many other units responding to this stimulus. Unit 1 was situated appropriately within a sea of label from the contralateral eye, just before the first lesion in Figure $18 B$ (white arrow). It was possible to test carefully the mixture of contra- and ipsilateral nerve inputs to nearby single units 2,3 , and 4 , and to another unit (unit 5) and a multiunit recording (unit 6) close to the second lesion. In each case, our standard procedure revealed unequivocal evidence for convergent excitation (Fig. 19).

The recording sites shown in Figure 19 are not spaced evenly along the electrode track. This is not because cells were only occasionally encountered, but rather the reverse: at most points there were too many spikes evoked by either stimulus. Various techniques were used to separate single units. For instance a double ipsilateral stimulus was chosen for unit 5 , not because a single stimulus was ineffective, but because in this instance a lower stimulus strength could be used and stimulation of nearby units avoided. Alternatively, convergent excitation was seen at many points along the track simply by means of multiunit recordings. Site 6 is an example. Here, the ipsilateral stimulus clearly facilitated the response to the contralateral nerve even though it did not evoke a response on its own.

A second electrode track in the E51 preparation gave the same result. Again, five additional single units all received convergent excitation from the two nerves.

Such convergence of excitation from the two nerves on to LGNd neurons was a dominant feature of all of the fetal preparations studicd. As shown in Table II, of the postsynaptic units tested in five preparations between E43 and E59, 31 of 34 units received simple bilateral excitation (Table II, column C). Only two units received excitation from one nerve and inhibition from the other (Table II, column B); it is significant that both of these units were recorded in the oldest preparationat E59. Comparison of results in the fetal and neonatal preparations shown in Table II reveals that LGN neurons in the two groups differ significantly from each other in the mixture of excitation and inhibition they receive from both optic nerves. Thus, during the period between $\mathrm{E} 43$ and birth a physiological shift in retinogeniculate connectivity takes place - a shift marked by a fall in the bilateral excitation of LGN neurons and by the appearance of inhibition from optic nerve stimulation.

\section{Discussion}

This in vitro electrophysiological study has shown that functional connections exist between the axons of retinal ganglion cells and the neurons of the cat's LGN by E40roughly 1 month before birth and as young an age as we have studied. We use the word "functional" rather than "functioning" intentionally here to emphasize the point that retinogeniculate connections are capable of function when tested, as we have done, by electrical stimulation in vitro. Anatomical studies indicate that, by E40, many retinal afferents from both eyes have already invaded the LGN - some as much as 1 week earlier - and are extensively intermixed with each other (Shatz, 1983). Intermixing persists until about 1 week later (E47), when the two sets of afferents begin to segregate progressively until the final adult-like laminar pattern of retinal input is achieved shortly after birth. Our microelectrode recordings here show that, throughout the prenatal period of intermixing, neurons of the LGN commonly receive convergent excitation from both optic nerves; not until very near birth does the adult-like inhibitory circuitry first emerge. Thus, the anatomical transformation from a mixed to a segregated state is accompanied by changes 

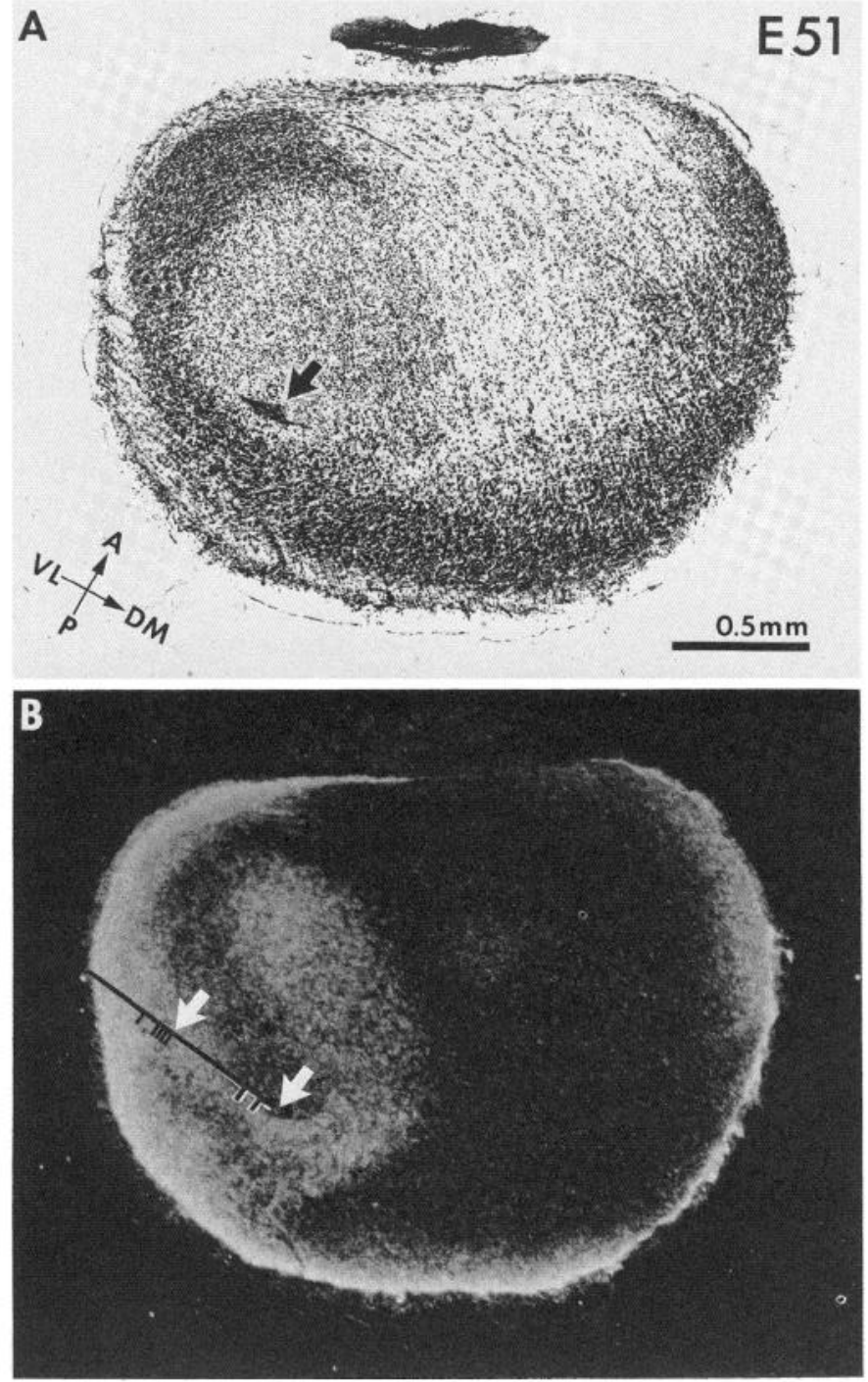

Figure 18. Reconstruction of an electrode track in an E51 preparation (to be viewed in conjunction with figure 19). $A$, Cresyl violet-stained section to show lesion (arrow) at end of track and the cytoarchitectonic definition of LGN. $B$, Darkfield autoradiograph of an adjacent section to show distribution of afferent terminals from the contralateral eye, which was injected with $\left[{ }^{3} \mathrm{H}\right]$ leucine $24 \mathrm{hr}$ before the in vitro experiment. The electrode track is drawn in, with sites of lesions indicated by white arrows and recording sites marked by black slashes.

in the snyaptic physiology of retinogeniculate connections.

Identity of neural elements recorded. As described under "Results," there was rarely a problem distinguishing presynaptic units (i.e., retinal afferent fibers) from postsynaptic units. The distinction was made on the basis of response properties rather than spike shape. (Spike shapes were often rather similar for pre- and postsynaptic units.) It is possible that some of the postsynaptic responses were recorded from efferent axons rather than neuronal somata, but this is unlikely for two reasons. First, single retinogeniculate afferents were rarely isolated in the optic tract; hence the afferent spikes we did record within the nucleus proper were most likely from action currents in the expanded membrane areas of terminals and preterminal branches. Second, in the neonatal animals where it was equally easy to record afferent units, there was a satisfying correspondence between the response properties and the laminar location of the postsynaptic units (Figs. 9 and 14). Thus, we think it highly probable that most postsynaptic unit recordings were from neuronal somata.

Our conclusions, in fact, are hardly affected by whether we were recording from somata or efferent axons, with one exception: we wished to know whether our recordings were from neurons of LGNd or LGNv in order to draw conclusions about the progressive loss of convergent excitation within the LGNd. Recordings from the LGNv or its efferent axons are complicated by the observation of Spear et al. (1977) that a much higher proportion of binocular excitation is found in the adult LGNv than in LGNd. However, we can be reasonably certain that the postsynaptic units recorded in the LGNd indeed represent the neurons of this nucleus because efferent LGNv axons are not known to traverse the LGNd. On the other hand, at present we have no idea whether the LGNd units recorded were principal cells, interneurons, or even cells not yet committed to either role.

Reliability of the in vitro observations. There are several good reasons to believe that the in vitro findings here provide reliable information concerning the sequence of prenatal development of synaptic connections. One is the good correlation between the results of our in vitro studies of the neonatal preparations and those from the many in vivo extracellular and intracellular studies of adult cat LGN unit responses to electrical stimulation of the optic nerves (Suzuki and Kato, 1966; Kato et al., 1971; Godfraind and Kelly, 1981; Jones, 1981; Lindström, 1982). Despite the fact that these two preparations (neonatal in vitro and adult in vivo) differ from each other not only in age, but also in connectivity (our preparation is no longer connected by afferents or efferents to the visual cortex or brainstem), more than half (13 of 21) of the postsynaptic units recorded extracellularly here received mixtures of excitation and inhibition from the two nerves similar to those found in vivo (Table II, columns B and D), indicating that at least some of the normal adult circuitry is alive and well in our preparation at birth.

The principal indicator of the state of the isolated preparation was the stability of the optic tract volley, which was well correlated with synaptic responsiveness in every preparation (see "Materials and Methods"). It is worth noting that the neonatal and oldest fetal preparations were actually the hardest to maintain in good condition. This was reflected both in volley stability and in the extent of necrosis seen histologically later on. Consequently, the majority of fetal preparations, possibly because of their smaller size and known greater resistance to anoxia (Duffy et al., 1975), are, if anything, even more likely than the neonatal examples to provide reliable information concerning the functional state of synaptic conections.

In view of these considerations, we think it highly likely that the universal absence of inhibition in the fetuses younger than E59, and the predominance of 


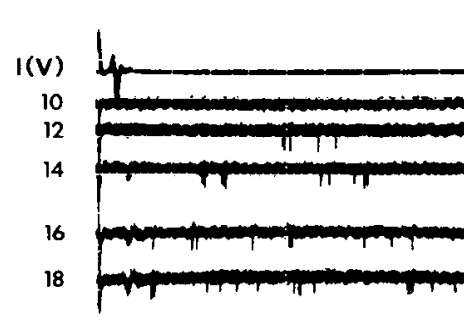

1
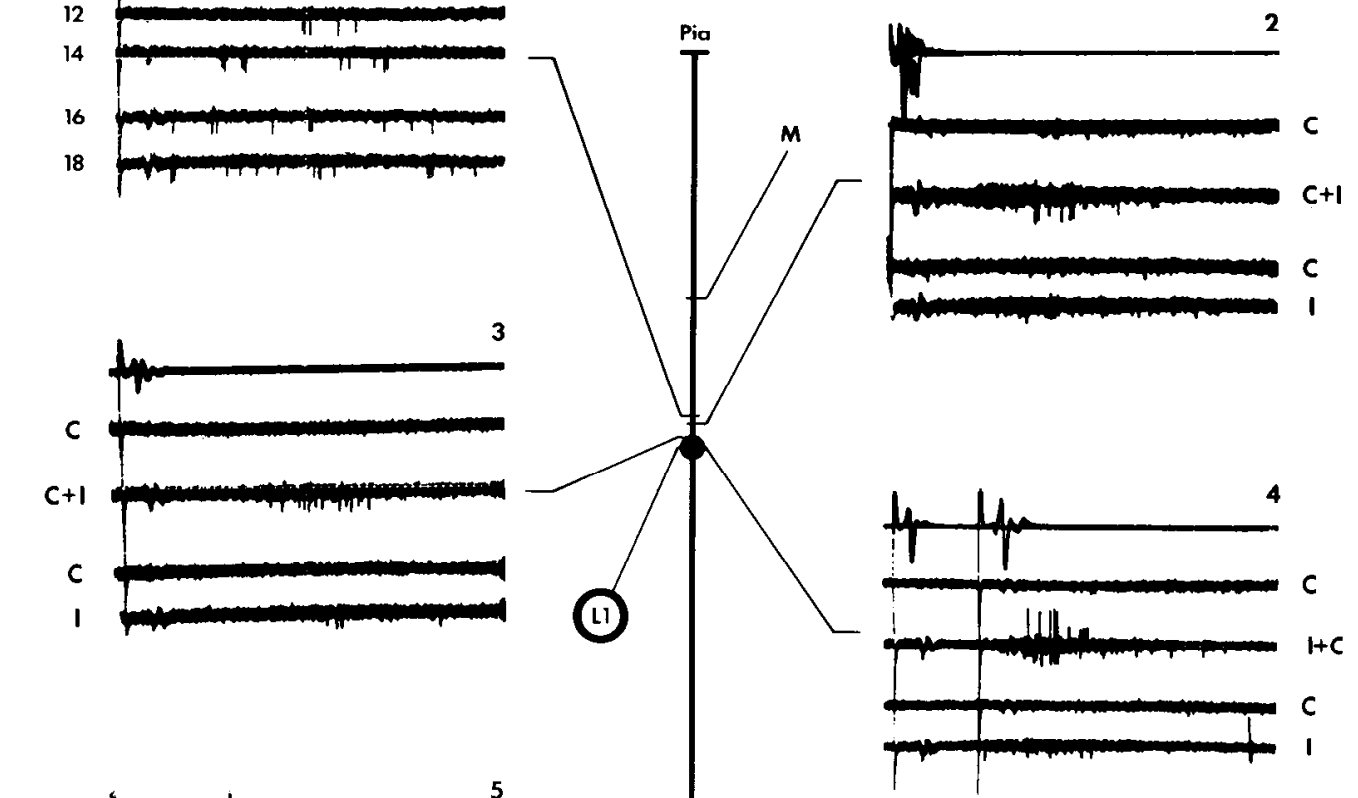

c
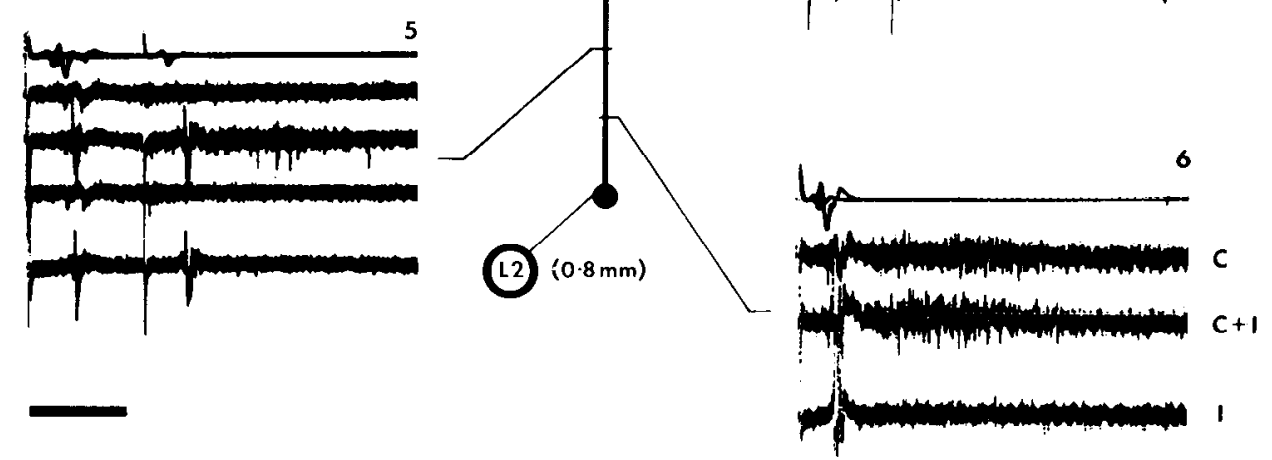

Figure 19. Properties of units recorded along the track shown in Figure 18. Positions along the line show the depth indicated on the electrode microdrive. Unit 1, Responses to ipsilateral stimulus at indicated voltages (five sweeps superimposed for each trace). Units 2 to 5 all show convergent excitation. The sequence of stimulation is as in Figure 7 (five sweeps superimposed for each trace.) Records for site 6 (multiunit recording) are similar in sequence except single sweeps with maximal stimuli were used and control stimulation of the contralateral nerve was omitted. $M$, site where multiunit postsynaptic responses were first observed; $L 1$ and $L 2$, lesions. Volleys were checked for stimulus spread with maximal stimuli and none was found at times of recording from sites $\mathrm{M}, 2$, and 5 . Time calibration, $50 \mathrm{msec}$ except for site 5 (33 msec).

inhibition by birth, can be taken as a real result rather than an artifact of the preparation. If so, then development of inhibition occurs rather late in fetal life and coincides in time with the conclusion of the segregation process. Late appearance of inhibition in fetal hippocampus (Schwartzkroin and Kunkel, 1982) and spinal cord (Saito, 1979) studied intracellularly in vitro has also been reported. Of course, in the present study we cannot totally rule out the presence of weak inhibition masked by strong convergent excitation in younger fetuses, but if inhibition is present, then it must be very weak indeed to go undetected with the same procedures that revealed combinations of excitation and inhibition in the neonatal preparations. We are also currently unable to identify the source of the inhibition. In the adult LGN there are at least two sources known: intrinsic interneurons which are excited by retinal afferents, and neurons of the perigeniculate nucleus which are excited by collaterals of the principal cells (Dubin and Cleland, 1977; Lindström, 1982). Of these two, cells of the perigeniculate nucleus are the least likely to be active in our preparations since, except for the lateral tip of the nucleus, this part of the thalamus is situated rather deep and it most likely to be in the necrotic region we observe histologically (cf. Fig. 9 and Shatz, 1983). Unfortunately, the long and variable latencies seen in these neonatal preparations make it impossible to use latency as a criterion to distinguish between these two sources, as is possible in the adult (Lindström, 1982).

In the youngest fetuses investigated (E39, E40) very few postsynaptic units were found, but by E47 they were plentiful and by E51 (Fig. 19) they seemed even more abundant. It is tempting to believe that this apparent increase in physiological responsiveness represents a real increase in synaptic effectiveness. This suggestion is supported by preliminary results of electron microscope 
TABLE II

Binocular convergent influences of optic nerve stimulation on single units in the LGNd

\begin{tabular}{ccccc}
\hline Age & $\begin{array}{c}\mathrm{A} \\
\text { Unilateral } \\
\text { Excitation } \\
\text { Only }\end{array}$ & $\begin{array}{c}\text { Unilateral } \\
\text { Excitation } \\
\text { plus } \\
\text { Inhibition }^{a}\end{array}$ & $\begin{array}{c}\mathrm{C} \\
\text { Bilateral } \\
\text { Excitation } \\
\text { Only }\end{array}$ & $\begin{array}{c}\mathrm{D} \\
\text { Bilateral } \\
\text { Excitation } \\
\text { plus } \\
\text { Inhibition }^{b}\end{array}$ \\
\hline $\begin{array}{c}\text { Neonates } \\
\text { P2 }^{c}\end{array}$ & 0 & 4 & 4 & \\
PO $^{c}$ & 1 & 5 & 3 & 2 \\
Fetal & & & & \\
E59 $^{c}$ & 0 & 2 & 3 & 0 \\
E54 $^{d}$ & 1 & 0 & 10 & 0 \\
E51 $^{c}$ & 0 & 0 & 10 & 0 \\
E49 $^{d}$ & 0 & 0 & 7 & 0 \\
E43 $^{c}$ & 0 & 0 & 1 & 0 \\
\hline
\end{tabular}

${ }^{a}$ Inhibition from one or both nerves.

${ }^{b}$ Inhibition from one nerve only.

${ }^{c}$ No interaction was present between the volleys even at maximal stimulus strength.

${ }^{d}$ Interaction was present, but only at maximal stimulus strengths.

which the retinogeniculate projection has been labeled at the electron microscopic level with horseradish peroxidase. By E43, diminutive synapses between identified retinal terminals and LGN neurons can be found, and these increase in number and size at progressively older fetal ages. Prior to E43, electron microscopic labeling efforts thus far have been unsuccessful; nevertheless, synapses (probably retinal in origin) can be found within the LGN as early as has been studied-E40 - a time when the in vitro recordings have revealed the existence of functional connections between retina and LGN.

Sampling. The ability to make successful recordings from the preparation we have used depends entirely on the fact that the optic tract and the IGN lie on the outside of the diencephalon and are thus accessible to $\mathrm{O}_{2}$ from the bathing medium. However, not all of the LGN could survive well in this way, as is demonstrated by the necrotic regions seen histologically. The question arises as to how much this restriction would have biased our sample of neurons. Our sample certainly contains fewer neurons from the deeper parts of the nucleus than from superficial regions; thus, for the fetus especially there was a bias toward neurons located in regions destined to become the C-layers (Shatz, 1983). However, without laminar boundaries for guidance, we cannot be certain exactly how many of the units should be assigned to future A-layers or C-layers. The situation is clarified somewhat with the autoradiographic labeling of one set of retinogeniculate afferents, an approach of most use in the older fetuses.

In view of this source of bias, strict quantitative comparisons between the physiological results reported here and the results of the earlier anatomical study of segregation of eye input within the LGN as a whole (Shatz, 1983) should not be made. It is worth noting, however, that in the present study the location of many electrode tracks near or in the future C-layers implies that recordings were frequently made from LGN regions in which the anatomy has shown that the inputs from the two eyes segregate relatively late (Shatz, 1983). (Indeed, a major goal was to study the functional implications of this anatomical intermixing.) Thus, the finding here that over $90 \%$ of the LGN units recorded in fetal preparations were driven by stimulation of both nerves is perfectly consistent with the sampling bias considered above.

The same source of bias may account in part for the relatively high proportion of bilaterally excited units (11 of 21; Table II, columns C and D) seen here in the neonatal preparations. In these cases our microelectrode penetrations were positioned unconventionally (but by necessity) to traverse the LGN from lateral to medial (Figs. 9 and 14). This arrangement increases significantly the percentage of the electrode track located near or within interlaminar regions where such units have been recorded in the adult LGN in vivo with dorsal-to-ventral electrode tracks (Sanderson et al., 1971). However, the proportion of such units would also be expected to be higher here than in the adult because anatomical methods have revealed that segregation of eye input, particularly at the interlaminar borders, is not quite complete by birth (Shatz, 1983, Fig. 13).

Finally, another source of bias comes from the use of extracellular recording in which probably only the most excitable neurons are accessible to study: in the youngest fetuses only a few neurons may reach threshold and fire an action potential; in the older ones, there were often too many neurons firing at high stimulus strengths to isolate single units successfully. Since we suspect that individual afferent axons in the fetuses produce only small synaptic potentials (see later), this may bias the sample to those neurons with the most afferent convergence on them. The question of convergent excitation from the two eyes, however, is not subject to the same bias (unless high convergence from one eye is related to the presence of input from the other) because our conditioning/testing procedure allowed us to observe subliminal effects. Moreover, the near-universal presence of convergent excitation in the fetus (including the multiunit recordings: Fig. 19) leads us to believe that some bias here would be of no consequence.

Nature of the synaptic connections. The most important difference between the observations on fetal preparations here and those made by others in the adult is that we observed convergent excitation from the two optic nerves onto most neurons of LGNd, whereas most cells in the adult LGN receive only monocular excitation but binocular inhibition. Now, as compared to the strong excitation seen in the adult (sometimes 1:1 firing from a single afferent) or occasionally in the neonatal preparations (cf. Fig. 11), the excitation we have observed in the fetus is weak, apparently requiring synchronous excitation from many input fibers. We base this conclusion on the observation that none of the postsynaptic units in the fetal preparations showed sharp, all-or-none firing thresholds with increasing stimulus strengths. In all cases, the strength of the response, assessed either by latency or by number of spikes per stimulus, gradually increased with increasing stimulus strength (e.g., Figs. 5, 13 , and 19 , unit 1 ). It is conceivable that such convergent excitation is present for most cells in the adult but has 
not been seen because it is weak and because it is masked by inhibition. However, this sort of weak monosynaptic excitation is ruled out (in the adult) in view of the total anatomical segregation of retinal input present in the adult LGN (Hickey and Guillery, 1973; Bowling and Michael, 1980; Sur and Sherman, 1982; Shatz, 1983). An anatomical substrate for an excitatory polysynaptic projection within the thalamus has also not been reported.

The conclusion we wish to draw, that the change from binocular excitation in the fetus to apparent monocular excitation in the adult involves the elimination of already functional synapses, thus depends upon the assumption that the excitation we have observed is monosynaptic. In general the latency of the excitation seen was equally short from the two nerves (e.g., Fig. 16), but these latencies are too long and variable to be of great help in this regard. The long time course of the excitation (e.g., Fig. 17), which is similar to other reports of fetal synaptic transmission (Naka, 1964; Saito, 1979), allows for many other mechanisms to be involved: chains of interneurons, interneurons firing in bursts, or even spread of excitation in a syncytial net. With the exception of the last possibility, however, these explanations require the synapses from second-order neurons to be as mature as those from the afferents in order to contribute to the polysynaptic pathway-an unlikely possibility in view of the late appearance of the presumed disynaptic inhibitory pathway seen here. Furthermore, although the possibility that LGN neurons are electrically coupled cannot be ruled out from the physiological evidence alone, electron microscopic evidence to date does not favor it, since gap junctions between LGN neurons have so far not been observed (C. J. Shatz and M. W. Siegel, manuscript in preparation). The most parsimonious explanation is that the excitation is monosynaptic, which allows us to conclude that the elimination of functional synapses indeed occurs. This conclusion is of course attractive because of the anatomical fact that the two sets of afferents are physically intermixed with each other during the period in which convergent excitation from both nerves is seen and because the physiological change from binocular to monocular excitation takes place at roughly the same time as the anatomical segregation of the afferent fibers (Shatz, 1983).

Physiological and developmental significance. A central goal of this study is to determine to what extent the process of segregation, as it occurs prenatally in the development of the cat's retinogeniculate connections, is similar to the many familiar examples of segregation of afferent inputs to other regions of the central and peripheral nervous systems (see Purves and Lichtman, 1980 , for review). Common to each postnatal example is the finding that the segregating inputs make functional synaptic connections with their target neurons throughout the process. The results of our in vitro experiments now permit us to state that the segregation of retinal afferents within the LGN according to eye input is no exception, despite the fact that it occurs almost entirely prenatally and therefore weeks before the visual pathway is put to the use for which it is ultimately designed. Indeed, given these results, we would expect that other sets of connections known from anatomical studies to segregate prenatally should also be functional at the time of segregation, regardless of whether they involve other components of the retinofugal pathway (Rakic, 1977; Williams and Chalupa, 1982) or even other systems within the mammalian CNS (Goldman-Rakic, 1981).

What we cannot determine from these in vitro experiments, however, is whether connections in utero between retinogeniculate afferents and LGN neurons are actually functioning spontaneously throughout the period of segregation as is the case when segregation occurs postnatally. Nor have we seen any spontaneous activity in vitro-either from the LGN neurons or from the afferent fibers. This is not particularly surprising in view of the fact that our preparations do not include the somata of the retinal ganglion cells which, at least in the adult, are known to be spontaneously active in the dark (Fuster et al., 1965; Rodieck, 1973). Nevertheless, we consider it highly likely that connections between retina and LGN are indeed functioning in utero precisely because functional connections can be demonstrated in vitro with electrical stimulation and hecause ultrastructural evidence of retinogeniculate synapses (Shatz et al., 1982) can be found throughout the period of segregation.

Perhaps the most important parallel to be drawn between the present results and those of previous postnatal studies is that the process of segregation is characterized physiologically in every instance by a reduction in the number of separate presynaptic inputs to individual target neurons. In the development of the cat's retinogeniculate pathway, this reduction is manifested in at least two ways. The first, the elimination of the convergent excitation from the two nerves, shows that the cat's retinogeniculate pathway is similar in its development to that of geniculocortical connections (Rakic, 1976; LcVay et al., 1978, 1980) and of the eighth nerve input to the cochlear nucleus (Jackson and Parks, 1982), in which it has been shown that an anatomical redistribution of axonal projections accompanies the physiological reduction in synaptic inputs from different sets of afferents.

The second manifestation was discussed above, where we suggested that neruons in the fetal LGN may receive relatively weak convergent excitatory inputs from many optic axons, whereas those in the neonatal or adult preparations may instead receive more powerful inputs from fewer axons. Analogous changes have been demonstrated directly by means of intracellular microelectrode recordings in a variety of other structures within the central and peripheral nervous systems (Brown et al., 1976; Purves and Lichtman, 1980; Mariani and Changeux, 1981a; Jackson and Parks, 1982). It is worth pointing out, however, that although much evidence favors a reduction in the number of individual axons supplying inputs to a particular postsynaptic neuron, this does not exclude the possibility that the total number of synapses present might actually increase with growth and development of the remaining axonal inputs and the dendritic trees of the LGN neurons. Observations of changes in the size and morphology of individual retinogeniculate afferents labeled with horseradish peroxidase suggest 
that this may indeed occur in the cat's visual system (Sretavan and Shatz, 1984).

In view of the many anatomical and physiological parallels between events associated with the prenatal segregation of retinogeniculate afferents and segregation as it occurs postnatally elsewhere in the vertebrate nervous system, it is highly likely that the underlying mechanism of segregation is a common one. In each instance it has been postulated that some sort of activity-related competition between the sets of segregating afferents for postsynaptic target neurons may occur (Stent, 1973; Changeux and Danchin, 1976; Purves and Lichtman, 1980; Stryker, 1981; Thompson, 1983). Indeed, a recent study of Archer et al. (1982) suggests that the final postnatal period of development of the cat's retinogeniculate pathway may depend on activity, since intraocular injections of tetrodotoxin alter the acquisition of normal receptive field properties within the LGN. The finding here that functional connections are in existence more than a month before they can be of any physiological use is strong independent evidence that this function has developmental significance. Without knowing that the synapses observed ultrastructurally can actually work, one might think that the competitive interactions take place solely by mechanisms such as contact, or spontaneous release (even if from synaptic vesicles) of trophic substances. However, because the neuronal properties commonly associated with the fully differentiated adult state (action potential generation and synaptic transmission) are present in the fetus, we believe that they are actually used in the process that brings about the adult pattern of connectivity, which, as far as binocularity is concerned, is largely present at birth. If so, then the process of segregation as it occurs prenatally is remarkably similar to that occurring postnatally, the principal difference being simply that the final outcome is susceptible to modification by experience if this process extends into postnatal life.

\section{References}

Archer, S. M., M. W. Dubin, and L. A. Stark (1982) Abnormal retinogeniculate connectivity in the absence of action potentials. Science 217: 743-745.

Bowling, D. B., and C. R. Michael (1980) Projection patterns of single physiologically characterized optic tract fibers in cat. Nature 286: 899-902.

Brown, M. C., J. K. S. Jansen, and D. Van Essen (1976) Polyneuronal innervation of skeletal muscle in new-born rats and its elimination during maturation. J. Physiol. (Lond.) 261: $387-422$.

Bunt, S. M., R. D. Lund, and P. W. Land (1983) Prenatal development of the optic projection in albino and hooded rats. Dev. Brain Res. 6: 149-168.

Changeux, J. -P., and A. Danchin (1976) Selective stabilization of developing synapses, a mechanism for the specification of neuronal networks. Nature 264: 705-712.

Cleland, B. G., M. W. Dubin, and W. R. Levick (1971) Simultaneous recording of input and output of lateral geniculate neurones. Nature New Biol. 231: 191-192.

Connors, B. W., B. R. Ransom, D. M. Kunis, and M. J. Gutnick (1982) Activity-dependent $\mathrm{K}+$ accumulation in the developing rat optic nerve. Science 216: 1341-1343.

Crepel, F., J. Mariani, and N. Delhaye-Bouchaud (1976) Evidence for a multiple innervation of Purkinje cells by climbing fibers in the immature rat cerebellum. J. Neurobiol. 7: 567578.

Crepel, F., S. S. Dhanjal, and J. Garthwaite (1981) Morphological and electrophysiological characteristics of rat cerebellar slices maintained in vitro. J. Physiol. (Lond.) 316: 127-138.

del Castillo, J., and B. Katz (1954) Statistical factors involved in neuromuscular facilitation and depression. J. Physiol. (Lond.) 124: 574-585.

Dingledinc, R. (1984) Brain Slices, Plenum Press, New York, in press.

Dubin, M. W., and B. G. Cleland (1977) Organization of visual inputs to interneurons of lateral geniculate nucleus of the cat. J. Neurophysiol. 40: 410-427.

Duffy, T. E., S. J. Kohle, and R. C. Vanucci (1975) Carbohydrate and energy metabolism in perinatal rat brain: Relation to survival in anoxia. J. Neurochem. 24: 271-276.

Fulton, B. P., R. Miledi, and 'T. 'Takahashi (1980) Electrical synapses between motoneurones in the spinal cord of the newborn rat. Proc. R. Soc. Lond. Biol. 208: 115-120.

Fuster, J. M., A. Herz, and O. D. Creutzfeldt (1965) Interval analysis of cell discharge in spontaneous and optically modulated activity in the visual system. Arch. Ital. Biol. 103: $159-177$.

Gasser, H. J. (1950) Unmedullated fibers originating in dorsal root ganglia. J. Gen. Physiol. 33: 651-690.

Godfraind, J. -M., and J. S. Kelly (1981) Intracellular recording from thin slices of the lateral geniculate nucleus of rats and cats. In Electrophysiology of Isolated Mammalian CNS Preparations, G. A. Kerkut and H. V. Wheal, eds., pp. 257-284, Academic Press, London.

Goldman-Rakic, P. S. (1981) Prenatal formation of cortical input and development of cytoarchitectonic compartments in the neostriatum of the rhesus monkey. J. Neurosci. 1: 721735 .

Guillery, R. W. (1969) The organization of synaptic interconnections in the laminae of the dorsal lateral geniculate nucleus of the cat. Z. Zellforsch. Mikrosk. Anat. 96: 1-38.

Hickey, T. L., and R. W. Guillery (1973) An autoradiographic study of retinogeniculate pathways in the cat and fox. J. Comp. Neurol. 156: 239-253.

Hubel, D. H. (1957) Tungsten microelectrode for recording from single units. Science 125: 549-550.

Jackson, H., and T. N. Parks (1982) Functional synapse elimination in the developing avian cochlear nucleus with simultaneous reduction in cochlear nerve axon branching. J. Neurosci. 2: 1736-1743.

Johnson, D. A., and D. Purves (1981) Postnatal reduction of neural unit size in the rabbit ciliary ganglion. J. Physiol. (Lond.) 318: 143-159.

Jones, E. G. (1981) Functional subdivision and synaptic organization of the mammalian thalamus. Int. Rev. Physiol. 25: 173-215.

Kalil, R. E., and G. Scott (1979) Development of retino-geniculate synapses in the dorsal lateral geniculate nucleus of the cat. Soc. Neurosci. Abstr. 5: 791.

Kato, H., M. Yamamoto, and H. Nakahama (1971) Intracellular recordings from the lateral geniculate neurones of cats. Jpn. J. Physiol. 21: 307-323.

Kirkwood, P. A., and C. J. Shatz (1983) Development of binocular inputs to dorsal lateral geniculate neurones in the fetal cat. J. Physiol. (Lond.) 336: 27-28P.

LeVay, S., M. P. Stryker, and C. J. Shatz (1978) Ocular dominance columns and their development in layer IV of the cat's visual cortex: A quantitative study. J. Comp. Neurol. 179: 223-244.

LeVay, S., T. N. Wiesel, and D. H. Hubel (1980) The development of ocular dominance columns in normal and visually deprived monkeys. J. Comp. Neurol. 191: 1-51. 
Lichtman, J. W., and D. Purves (1980) The elimination of redundant preganglionic innervation to hamster sympathetic ganglion cells in early postnatal life. J. Physiol. (Lond.) 301: 213-228.

Linden, D. C., R. W. Guillery, and J. Cucchiaro (1981) The dorsal lateral geniculate of the normal ferret and its postnatal development. J. Comp. Neurol. 203: 189-211.

Lindstrom, S. (1982) Synaptic organization of inhibitory pathways to principal cells in the lateral geniculate nucleus of the cat. Brain Res. 234: 447-453.

Mariani, J., and J. -P. Changeux (1981a) Ontogenesis of olivocerebellar relationships. I. Studies by intracellular recordings of multiple innervation of Purkinje cells by climbing fibers in the developing rat cerebellum. J. Neurosci. 1: 696702.

Mariani, J., and J. -P. Changeux (1981b) Ontogenesis of olivocerebellar relationships. II. Spontaneous activity of inferior olivary neurons and climbing fiber-mediated activity of cerebellar Purkinje cells in developing rats. J. Neurosci. 1: 703-709.

Mason, C. A. (1982) Development of terminal arbors of retinogeniculate axons in the kitten. II. Electron microscopical observations. Neuroscience 3: 561-582.

Naka, K. -I. (1964) Electrophysiology of the fetal spinal cord. I. Action potentials of the motoneuron. J. Gen. Physiol. 47: 1003-1022.

Ono, T., and W. K. Noel (1973) Characteristics of P- and Icells of the cat's lateral geniculate body. Vision Res. 13: 639646.

Purves, D., and J. W. Lichtman (1980) Elimination of synapses in the developing nervous system. Science 210: 153-157.

Rakic, P. (1976) Prenatal genesis of connections subserving ocular dominance in the rhesus monkey. Nature 261: 467471.

Rakic, P. (1977) Prenatal devlopment of the visual system in rhesus monkey. Philos. Trans. R. Soc. Lond. 278: 245-260.

Rodieck, R. W. (1973) The Vertebrate Retina, pp. 600-603, W. H. Freeman and Co., San Francisco.

Saito, K. (1979) Development of spinal reflexes in the rat fetus studied in vitro. J. Physiol. (Lond.) 294: 581-594.

Sanderson, K. J., P. O. Bishop, and I. Darian-Smith (1971) The properties of binocular receptive fields of lateral geniculate neurons. Exp. Brain Res. 13: 178-207.

Schwartzkroin, P. A. (1981) To slice or not to slice. In Electrophysiology of Isolated Mammalian CNS Preparations, G. A. Kerkut and H. V. Wheal, eds., pp. 15-50, Academic Press, London.

Schwartzkroin, P. A., and D. D. Kunkel (1982) Electrophysiol- ogy and morphology of the developing hippocampus in fetal rabbits. J. Neurosci. 2: 448-462.

Shatz, C. J. (1977) Abnormal interhemispheric connections in the visual system of Boston Siamese cats: A physiological study. J. Comp. Neurol. 171: 229-246.

Shatz, C. J. (1983) The prenatal development of the cat's retinogeniculate pathway. J. Neurosci. 3: 482-499.

Shatz, C. J., P. A. Kirkwood, and M. W. Siegel (1982) Functional retinogeniculate synapses in fetal cats. Soc. Neurosci. Abstr. 8: 815.

Singer, W., and H. D. Lux (1973) Presynaptic depolarization and extracellular potassium in the cat lateral geniculate nucleus. Brain Res. 64: 17-33.

So, K. -F., G. E. Schneider, and D. O. Frost (1978) Postnatal development of retinal projections to the lateral geniculate body in Syrian hamsters. Brain Res. 142: 343-352.

Spear, D. D., D. C. Smith, and L. L. Williams (1977) Visual receptive field properties of single neurons in cat's ventral lateral geniculate nucleus. J. Neurophysiol. 40: 390-409.

Sretavan, D. W., and C. J. Shatz (1984) Prenatal development of retinogeniculate axons during the period of segregation. Nature, in press.

Stent, G. S. (1973) Physiological mechanism for Hebb's postulate of learning. Proc. Natl. Acad. Sci. U. S. A. 70: 9971001.

Stryker, M. P. (1981) Late segregation of geniculate afferents to the cat's visual cortex after recovery from binocular impulse blockade. Soc. Neurosci. Abstr. 7: 842 .

Sur, M., and S. M. Sherman (1982) retinogeniculate terminations in cats: Morphological differences between $\mathrm{X}$ and $\mathrm{Y}$ cell axons. Science 218: 389-391.

Suzuki, H., and H. Kato (1966) Binocular interaction at cat's lateral geniculate body. J. Neurophysiol. 29: 909-920.

Thompson, W. (1983) Synapse elimination in neonatal rat muscle is sensitive to pattern of muscle use. Nature 302: 614616.

Williams, R. W. and Chalupa, L. M. (1982) Prenatal development of retinocollicular projections in the cat: An anterograde tracer transport study. J. Neurosci. 2: 604-622.

Williams, R. W., M. J. Bastiani, and L. M. Chalupa (1983) Loss of axons in the cat optic nerve following fetal unilateral enucleation: An electron microscopic analysis. J. Neurosci. 3: 133-144.

Winfield, D. A., and T. P. S. Powell (1980) An electronmicroscopical study of the postnatal development of the lateral geniculate nucleus in the normal kitten and after eyelid suture. Proc. R. Soc. Lond. Biol. 210: 197-210. 